\title{
Space-Time Trellis and Space-Time Block Coding Versus Adaptive Modulation and Coding Aided OFDM for Wideband Channels
}

\author{
Tong-Hooi Liew and Lajos Hanzo, Fellow, IEEE
}

\begin{abstract}
The achievable performance of channel coded spacetime trellis (STT) codes and space-time block (STB) codes transmitted over wideband channels is studied in the context of schemes having an effective throughput of 2 bits/symbol (BPS) and 3 BPS. At high implementational complexities, the best performance was typically provided by Alamouti's unity-rate $G_{2}$ code in both the 2-BPS and 3-BPS scenarios. However, if a low complexity implementation is sought, the 3-BPS 8PSK space-time trellis code outperfoms the $G_{2}$ code. The $G_{2}$ space-time block code is also combined with symbol-by-symbol adaptive orthogonal frequency division multiplex (AOFDM) modems and turbo convolutional channel codecs for enhancing the system's performance. It was concluded that upon exploiting the diversity effect of the $G_{2}$ space-time block code, the channel-induced fading effects are mitigated, and therefore, the benefits of adaptive modulation erode. In other words, once the time- and frequency-domain fades of the wideband channel have been counteracted by the diversity-aided $G_{2}$ code, the benefits of adaptive modulation erode, and hence, it is sufficient to employ fixed-mode modems. Therefore, the low-complexity approach of mitigating the effects of fading can be viewed as employing a single-transmitter, single-receiver-based AOFDM modem. By contrast, it is sufficient to employ fixed-mode OFDM modems when the added complexity of a two-transmitter $G_{2}$ scheme is affordable.
\end{abstract}

Index Terms-Adaptive modulation and coding, HSDPA, OTDM, STBC, STTC.

\section{INTRODUCTION}

$\mathbf{S}$ PACE-TIME block (STB) codes were proposed in the seminal contributions of Alamouti [1], as well as Tarokh et al. [2], [3], while their achievable performance was investigated over perfectly interleaved, nondispersive Rayleigh fading channels, for example, in [4] and [5]. Furthermore, space-time trellis (STT) codes [6]-[11] were proposed by Tarokh et al. which incorporate jointly designed channel coding, modulation, transmit diversity, and optional receiver diversity. The performance criteria for designing space-time trellis codes were outlined in [6], under the assumption that the channel is fading slowly and that the fading is frequency nonselective. It was shown in [6] that the system's performance is determined by matrices constructed from pairs of distinct code sequences. Both the diversity gain and coding gain of the codes are determined

Manuscript received December 23, 2003; revised March 6, 2005. The review of this paper was coordinated by Prof. J. Shea.

The authors are with the Department of Electronics and Computer Science, University of Southampton, Southampton, SO17 1BJ U.K. (e-mail: lh@ecs.soton.ac.uk).

Digital Object Identifier 10.1109/TVT.2005.861174 by the minimum rank and the minimum determinant [6], [12] of the matrices, respectively. The results were then also extended to fast fading channels. The STT codes proposed in [6] provide the best tradeoff between data rate, diversity advantage, and trellis complexity.

The performance of both STT and STB codes over narrowband Rayleigh fading channels was investigated by numerous researchers [1], [3], [6], [10], [13]. The investigation of space-time codes was then also extended to the class of practical wideband fading channels. The effect of multiple propagation paths on the performance of STT codes was studied in [11] for transmission over slowly varying Rayleigh fading channels. It was shown in [11] that the presence of multiple paths does not decrease the diversity order guaranteed by the design criteria used to construct the STT codes. The evidence provided in [11] was then also extended to rapidly fading dispersive and nondispersive channels. As a further performance improvement, turbo equalization was employed in [14] in order to mitigate the effects dispersive channels. However space-time coded turbo equalization involved high complexity. In addressing the complexity issues, Bauch et al. [15] derived finite length multiple-input multiple-output (MIMO) channel filters and used them as prefilters for turbo equalizers. These prefilters significantly reduce the number of turbo equalizer states and, hence, mitigate the decoding complexity. As an alternative solution, the effect of intersymbol interference (ISI) could be eliminated by employing orthogonal frequency division multiplexing (OFDM) [16], [17]. Employing a system using STT-coded OFDM is attractive, since the decoding complexity reduced, as demonstrated by the recent surge in research interest [13], [18]-[20]. In [13], [18] and [20], nonbinary Reed-Solomon (RS) codes were employed in the STT-coded OFDM systems to improve their performance.

Similarly, the performance of STB codes was also investigated over frequency-selective Rayleigh fading channels. In [21], a MIMO equalizer was utilized for equalizing the dispersive multipath channels. Furthermore, the advantages of OFDM were also exploited in STB-coded systems [13], [22], [23].

In this contribution, we assume that the readers are familiar with the encoding and decoding processes of both STB and STT codes [5]-[11]. This paper does not design new STT codes, block codes, error correction codes, or adaptive modulation schemes. The contribution of this treatise is rather that of providing system designers with a performance versus implementational complexity-type technology road-map, the best 
design choices at a given system complexity and throughput in various propagation scenarios.

The structure of the paper is as follows. In Section II, our system model is introduced, which enables the comparison of STT and STB codes transmitted over wideband fading channels. Our simulation results are then provided in Section III. We continue our investigations by employing space-time coded adaptive modulation-based OFDM in Section IV. Finally, we conclude our discourse in Section V.

\section{SPACE-Time Coded Transmission Over WIDEBAND CHANNELS}

We now investigate the performance of space-time codes over dispersive wideband fading channels. As mentioned in Section I, Bauch's approach [14], [15] of using turbo equalization for mitigating the ISI exhibits considerable complexity. Hence, we argued that using space-time-coded OFDM constitutes an attractive approach of communicating over dispersive wireless channels, since the associated decoding complexity is significantly lower. Therefore, in this contribution, OFDM is employed for mitigating the effects of dispersive channels.

It is widely recognized that STT codes [6] perform well at a cost of high complexity. However, Alamouti's $\mathbf{G}_{2}$ STB code [1] could be invoked instead of STT codes. The space-time block code $\mathbf{G}_{2}$ is appealing in terms of its simplicity, although there is a slight loss in performance. Recall that it is vital for the $\mathbf{G}_{2}$ code that the channel's attenuation and phase rotation remains the same for its two consecutive time slots [6]. However, in order to facilitate transmissions over dispersive or wideband channels, in this contribution, we invoked OFDM, and instead of mapping the signals $x_{1}, x_{2}$ to two consecutive time slots, we mapped them to two adjacent OFDM subcarriers.

Furthermore, we concatenated the space-time block code $\mathbf{G}_{2}$ with turbo convolutional (TC) codes in order to improve the performance of the system. The family of TC codes was favored, because it was shown in [5] that in a space-time coded context, TC codes achieve an attractive coding gain at moderate complexity when compared to convolutional codes, turbo $\mathrm{BCH}$ codes, trellis-coded modulation, and turbo trellis-coded modulation. The performance of concatenated STB codes and TC codes will then be compared to that of STT codes. Conventionally, Reed-Solomon [5] (RS) codes have been employed in conjunction with the STT codes [13], [18], [20] for improving the performance of the system. In our forthcoming discussion, we will concentrate on comparing the performance of STB and STT codes in conjunction with various channel coders.

\section{A. System Overview}

Fig. 1 shows the schematic of the system employed in our performance study. At the transmitter, the information source generates random information data bits. The information bits are then encoded by TC codes, RS codes, or left uncoded. The coded or uncoded bits are then channel interleaved, as shown in Fig. 1. The output bits of the channel interleaver are then passed to the STT or STB encoder. We will investigate a number of STT codes proposed by Tarokh et al. [6], where the associated trellis

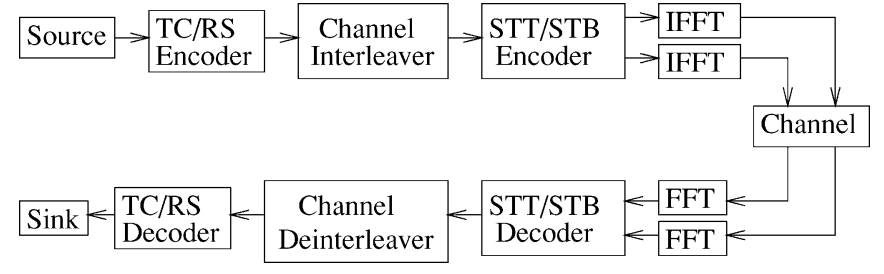

Fig. 1. System overview.

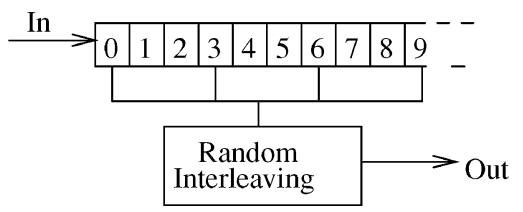

Fig. 2. Random separation-based interleaving.

state transition diagrams are shown, for example, in [5, Fig. 11.6-11.12]. The modulation schemes employed are 4PSK as well as 8PSK. On the other hand, from the family of STB codes, only Alamouti's $\mathbf{G}_{2}$ code is employed in the system, since it was shown in [5] and [24] that the best performance is achieved by concatenating the unity-rate STB code $\mathbf{G}_{2}$ with TC codes rather than its lower rate counterparts using a higher number of antennas. Furthermore, although turbo BCH codes, trellis coded modulation (TCM) and convolution codes were also used in the investigations of [5], [24]; again, the best performer was the TC code at a given implementational complexity. For convenience, the transmission matrix of the space-time block code $\mathbf{G}_{2}$ is reproduced here as

$$
\mathbf{G}_{2}=\left(\begin{array}{cc}
x_{1} & x_{2} \\
-\bar{x}_{2} & \bar{x}_{1}
\end{array}\right)
$$

where, again, $\bar{x}_{2}, \bar{x}_{1}$ indicate the conjugate complex versions of $x_{2}, x_{1}$. Different modulation schemes may be employed [25], such as binary phase shift keying (BPSK), quadrature phase shift keying (QPSK), 16-level quadrature amplitude modulation (16QAM), and 64-level quadrature amplitude modulation (64QAM). Gray mapping of the bits to symbols was applied, and this resulted in different protection classes in higher order modulation schemes [16], [17]. The mapping of the data bits and parity bits of the TC encoder was chosen such that it yielded the best achievable performance along with the application of the random separation-based channel interleaver [26].

Fig. 2 shows an example of the random separation-based interleaving employed. If, for example, 8-PSK modulation is used, then 3 bits per modulation constellation point are transmitted. Hence, for every 3-bit spaced position, the bits will be randomly interleaved. For an example, in Fig. 2 we randomly interleaved the bit positions $0,3,6,9, \ldots$ Similarly, bit positions $1,4,7, \ldots$, and $2,5,8, \ldots$ were also randomly interleaved. The objective of random separation-based interleaving is to randomly interleave the bits within the same protection class of the modulated symbol, while at the same time avoiding mixing the bits belonging to different protection classes. 
TABLE I

PARAMETERS OF THE SPACE-TIME TRELlis CODES USED

\begin{tabular}{|c|c|c|c|c|c|}
\hline \multirow{2}{*}{$\begin{array}{c}\text { Modulation } \\
\text { scheme }\end{array}$} & BPS & $\begin{array}{c}\text { Decoding } \\
\text { algorithm }\end{array}$ & $\begin{array}{c}\text { No. of } \\
\text { states }\end{array}$ & $\begin{array}{c}\text { No. of } \\
\text { transmitters }\end{array}$ & $\begin{array}{c}\text { No. of } \\
\text { termination } \\
\text { symbols }\end{array}$ \\
\hline 4PSK & 2 & VA & 4 & 2 & 1 \\
\cline { 4 - 6 } & & & 8 & 2 & 2 \\
\cline { 4 - 6 } & & & 16 & 2 & 2 \\
\hline 8PSK & \multirow{2}{*}{3} & \multirow{2}{*}{ VA } & 8 & 2 & 3 \\
\cline { 4 - 6 } & & & 16 & 2 & 2 \\
\cline { 4 - 6 } & & & 32 & 2 & 2 \\
\hline
\end{tabular}

The output of the space-time encoder was then OFDM [16], [17] modulated and transmitted by the corresponding antenna. Again, instead of mapping the signals $x_{1}, x_{2}$ to two consecutive time slots having identical channel attenuation and phase rotation as in [6], we mapped them to two adjacent OFDM subcarriers. The number of transmit antennas was fixed to two, while the number of receive antennas constituted an adjustable design parameter. Dispersive wideband channels were used, and the associated channels' profiles will be discussed later.

At the receiver, the signal of each receive antenna is OFDM demodulated. The demodulated signals of the receiver antennas are then fed to the STT or STB decoder. The space-time decoders apply the MAP [27] or Log-MAP [28], [29] decoding algorithms for providing soft outputs for the channel decoders. If no channel codecs are employed in the system, the space-time decoders apply the Viterbi algorithm (VA) [5], [6], [30], which gives similar performance to the MAP decoder [5] (VA) at a lower complexity. The decoded bits are finally passed to the sink for the calculation of the bit error rate (BER) or frame error rate (FER).

\section{B. Space-Time and Channel Codec Parameters}

In Fig. 1, we have given an overview of the system studied. In this section, we present the parameters of the space-time codes and the channel codecs employed in the system, which are summarized in Table I. On the other hand, from the family of STB codes, only Alamouti's $\mathbf{G}_{2}$ code is employed, since we have shown in [24] that the best performance in the set of investigated schemes was obtained by concatenating the STB code $\mathbf{G}_{2}$ with TC codes. The transmission matrix of the code is shown in (1), while the number of transmitters used by the STB code $\mathbf{G}_{2}$ is two, which is identical to the number of transmitters in the STT codes shown in Table I.

We now consider the TC channel codes used. We will concentrate on using the simple half-rate $\mathrm{TC}(2,1,3)$ code. Its associated parameters are shown in Table II. As seen in Table III, different modulation schemes are employed in conjunction with the concatenated STB code $\mathbf{G}_{2}$ and the TC $(2,1,3)$ code. Since the half-rate $\mathrm{TC}(2,1,3)$ code is employed, higher order modulation schemes such as 16QAM and 64QAM were chosen so that the throughput of the system remained the same as that of the system
TABLE II

ASSOCIATED PARAMETERS OF THE TC $(2,1,3)$ CODE

\begin{tabular}{|c|c|c|c|c|c|}
\hline Code & $\begin{array}{c}\text { Octal } \\
\text { generator } \\
\text { polynomial }\end{array}$ & $\begin{array}{c}\text { No. } \\
\text { of } \\
\text { states }\end{array}$ & $\begin{array}{c}\text { Decoding } \\
\text { algorithm }\end{array}$ & $\begin{array}{c}\text { Puncturing } \\
\text { pattern }\end{array}$ & $\begin{array}{c}\text { of } \\
\text { iterations }\end{array}$ \\
\hline $\mathrm{TC}(2,1,3)$ & 7,5 & 4 & Log-MAP & 10,01 & 8 \\
\hline
\end{tabular}

TABLE III

Simulation Parameters Associated With the TC $(2,1,3)$ Code

\begin{tabular}{|c|c|c|c|c|c|}
\hline Code & $\begin{array}{c}\text { Code } \\
\text { Rate } \\
R\end{array}$ & $\begin{array}{l}\text { Modula- } \\
\text { tion } \\
\text { Mode }\end{array}$ & BPS & $\begin{array}{c}\text { Random } \\
\text { turbo } \\
\text { interleaver } \\
\text { depth }\end{array}$ & $\begin{array}{c}\text { Random } \\
\text { separation } \\
\text { interleaver } \\
\text { depth }\end{array}$ \\
\hline & & \multicolumn{4}{|c|}{128 carriers } \\
\hline \multirow[t]{5}{*}{$\mathrm{TC}(2,1,3)$} & \multirow[t]{5}{*}{0.50} & 16QAM & 2 & 256 & 512 \\
\hline & & 64QAM & 3 & 384 & 768 \\
\hline & & \multicolumn{4}{|c|}{512 carriers } \\
\hline & & QPSK & 1 & 512 & 1024 \\
\hline & & 16QAM & 2 & 1024 & 2048 \\
\hline
\end{tabular}

employing the STT codes without channel coding. It is widely recognized that the performance of TC codes improves upon increasing the turbo interleaver size, and near-optimum performance can be achieved using large interleaver sizes exceeding 10000 bits. However, this performance gain is achieved at a cost of high latency, which is impractical for a delay-sensitive real-time system. On the other hand, STT codes offer impressive coding gains [6] at low latency. The decoding of the STT codes is carried out on a transmission burst-by-burst basis. In order to make a fair comparison between the systems investigated, the turbo interleaver size was chosen such that all the coded bits were hosted by one transmission burst. This enables burstby-burst turbo decoding at the receiver. Since we employ an OFDM modem, latency may also be imposed by a high number of subcarriers in an OFDM symbol. Therefore, the turbo interleaver size was increased as the number of subcarriers increased in our investigations. In Table III, we summarized the modulation schemes and interleaver sizes used for different numbers of OFDM subcarriers in the system. The random separation based channel interleaver of [5] and [26] was used. The mapping of the data bits and parity bits into different protection classes of the higher order modulation scheme was carried out such that the best possible performance was attained. Again, this issue was addressed in [5] and [26].

RS codes [5] were employed in conjunction with the STT codes. Hard decision decoding was utilized, and the coding parameters of the RS codes employed are summarized in Table IV.

We note, however, that the achievable performance of RS codes may be further improved in conjunction with errors-anderasures decoding, since the decoder is typically capable of correcting twice as many erasures as errors [31]. ${ }^{1}$

\footnotetext{
${ }^{1}$ The authors would like to thank Dr. J. Shea for his valuable related comment.
} 
TABLE IV

CODING PARAMETERS OF THE REED-SOLOMON CODES EMPLOYED

\begin{tabular}{|l|c|c|c|}
\hline Code & $\begin{array}{c}\text { Galois } \\
\text { Field }\end{array}$ & Rate & $\begin{array}{c}\text { Correctable } \\
\text { symbol errors }\end{array}$ \\
\hline RS(105,51) & $2^{10}$ & 0.49 & 27 \\
\hline RS(153,102) & $2^{10}$ & 0.67 & 25 \\
\hline
\end{tabular}

\section{Complexity Issues}

In this section, we will address the implementational complexity issues of the systems studied. We will, however, focus mainly on the relative complexity of the systems, rather than attempting to quantify their exact complexity. In order to simplify our comparative study, several assumptions are stipulated. In our simplified approach, the estimated complexity of the system is deemed to depend only on that of the STT decoder and turbo decoder. In other words, the complexity associated with the modulator, demodulator, STT encoder, and decoder, as well as that of the STT encoder and turbo encoder, are assumed to be insignificant compared to the complexity of STT decoder and turbo decoder.

In [5] and [24], we have detailed our complexity estimates, where the estimated complexity of the TC decoder is assumed to depend purely on the number of trellis transitions per information data bit. This simple estimated complexity measure was also used in as the basis of our comparisons.

In [5, Fig. 11.6-11.12], we have shown the state diagrams of the 4PSK and 8PSK STT codes used here. From these state diagrams, we can see that the number of trellis transitions leaving each state is equivalent to $2^{\mathrm{BPS}}$, where BPS denotes the number of transmitted bits per modulation symbol. Since the number of information bits is equal to BPS, we can approximate the complexity of the STT decoder as

$$
\begin{aligned}
\operatorname{comp}\{\mathrm{STT}\} & =\frac{2^{\mathrm{BPS}} \times \text { No. of States }}{\text { BPS }} \\
& =2^{\text {BPS-1}} \times \text { No. of States. }
\end{aligned}
$$

Applying (2) and assuming that the Viterbi decoding algorithm was employed, we tabulated the approximated complexities of the STT decoder in Table V.

\section{Simulation Results}

In this section, we will present our simulation results characterizing the OFDM-based system investigated. As mentioned previously, we will investigate the system's performance over dispersive wideband Rayleigh fading channels. We will commence our investigations using a simple two-ray channel impulse response (CIR) having equal tap weights, followed by a more realistic wireless asynchronous transfer mode (WATM) channel [16], [17]. In the CIR of the two-ray model, the reflected path has the same amplitude as the line of sight (LOS) path, although arriving $5 \mu$ s later. However, in our later simulations, we also present performance results for transmissions over two-ray channels separated by various delay spreads, rang-
TABLE V

ESTIMATED COMPLEXITY OF THE STT DECODERS

\begin{tabular}{|c|c|c|c|}
\hline $\begin{array}{c}\text { Modulation } \\
\text { scheme }\end{array}$ & BPS & $\begin{array}{c}\text { No. of } \\
\text { states }\end{array}$ & Complexity \\
\hline \multirow{2}{*}{4 PSK } & 2 & 4 & 8 \\
\cline { 3 - 4 } & & 8 & 16 \\
\cline { 3 - 4 } & & 16 & 32 \\
\cline { 3 - 4 } & & 32 & 64 \\
\hline \multirow{2}{*}{8 PSK } & 3 & 8 & 21.33 \\
\cline { 3 - 4 } & & 16 & 42.67 \\
\cline { 3 - 4 } & & 32 & 85.33 \\
\hline
\end{tabular}

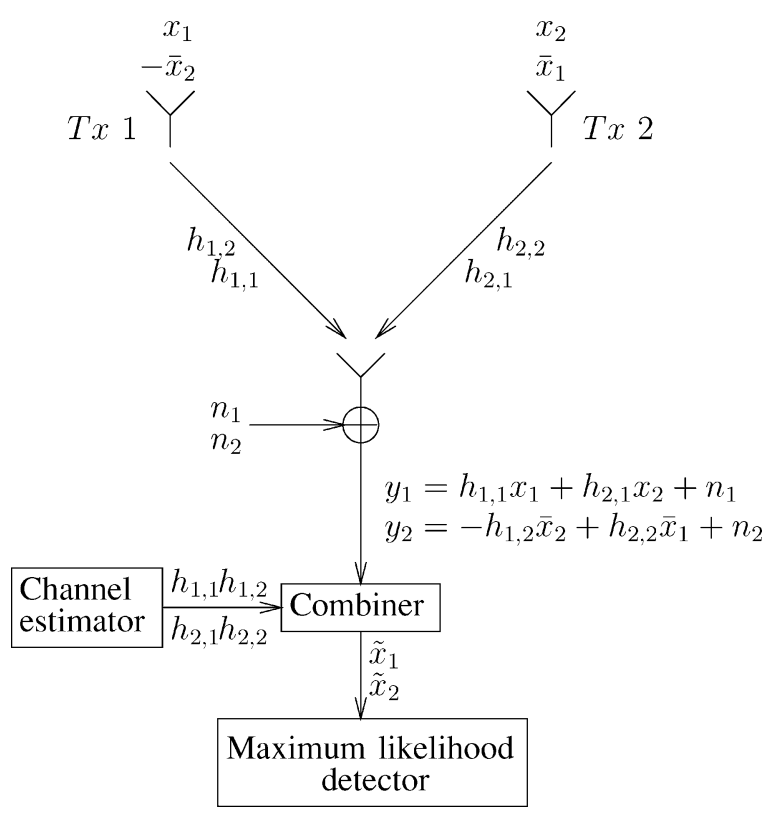

Fig. 3. Baseband representation of the simple twin transmitter STB code $\mathbf{G}_{2}$ of (1) using one receiver over varying fading conditions.

ing up to $40 \mu \mathrm{s}$. Jakes' model [32] was adapted for modeling the fading channels. Initially, a 128-subcarrier OFDM symbol was employed, having a guard period of $40 \mu \mathrm{s}$. The guard period of $40 \mu \mathrm{s}$ corresponded to a cyclic extension of 32 samples, which was sufficiently long for overcoming the inter-OFDM symbol interference imposed by the dispersive channel's memory. We assumed furthermore that the average signal power received from each transmitter antenna was the same, that all multipath components undergo independent Rayleigh fading, and that the receiver has a perfect knowledge of the CIR. We note that the above assumptions are aften unrealistic, yielding the best-case performance, nonetheless facilitating the performance comparison of the various techniques under identical circumstances.

\section{A. Space-Time Coding Comparison-Throughput of 2 BPS and 3 BPS}

In Fig. 4, we show our FER performance comparison between 4PSK STT codes and the STB code $\mathbf{G}_{2}$ concatenated with the $\mathrm{TC}(2,1,3)$ code using one receiver and the 128 -subcarrier 


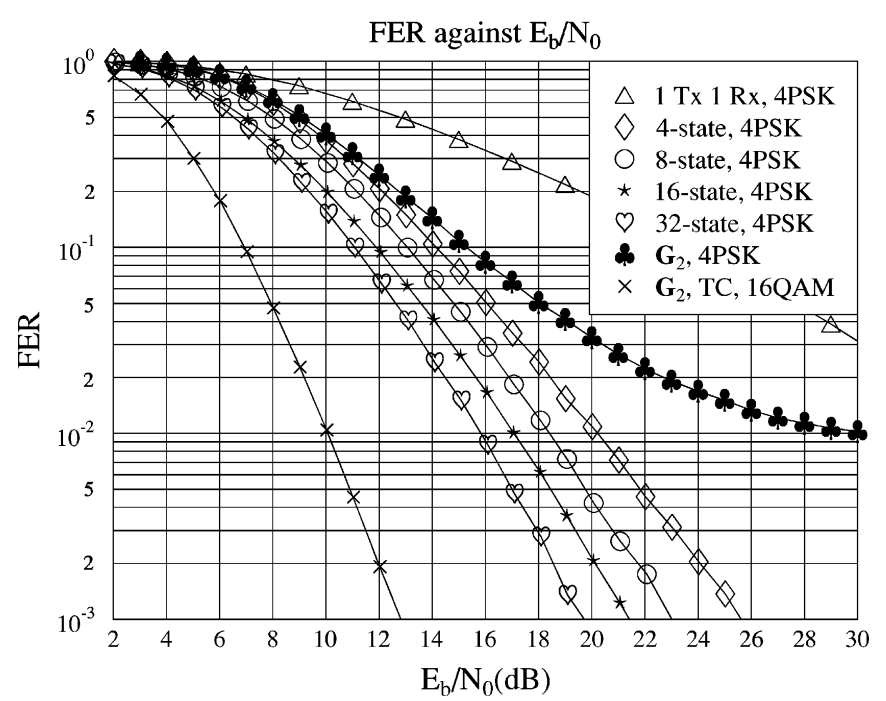

Fig. 4. FER performance comparison between various 4PSK STT codes and the STB code $\mathbf{G}_{2}$ concatenated with the $\mathrm{TC}(2,1,3)$ code using one receiver and the 128-subcarrier OFDM modem over a channel having a CIR characterized by two equal-power rays separated by a delay spread of $5 \mu \mathrm{s}$. The maximum Doppler frequency was $200 \mathrm{~Hz}$. The effective throughput was $2 \mathrm{BPS}$, and the coding parameters are shown in Tables I-III.

OFDM modem. The CIR had two equal power rays separated by a delay spread of $5 \mu \mathrm{s}$, and the maximum Doppler frequency was $200 \mathrm{~Hz}$. The $\mathrm{TC}(2,1,3)$ code is a half-rate code, and hence, 16QAM was employed in order to support the same 2 BPS throughput as the 4PSK space-time trellis codes using no channel codes. We can clearly see that at FER $=10^{-3}$, the performance of the concatenated scheme is at least $7 \mathrm{~dB}$ better than that of then STT codes.

The performance of the STB code $\mathbf{G}_{2}$ without $\mathrm{TC}(2,1,3)$ channel coding is also shown in Fig. 4. It can be seen in the figure that without channel coding, the STB code $\mathbf{G}_{2}$ does not perform well, exhibiting a residual BER, which is a consequence of the channel-induced intersubcarrier interference of the OFDM modem, rendering the channel transfer factors of the $\mathbf{G}_{2}$ code's $x_{1}$ and $x_{2}$ components different. This issue will be addressed in more depth during our subsequent discourse. Moreover, at high $E_{b} / N_{0}$ values, the performance of the single-transmitter, single-receiver system is better than that of the STB code $\mathbf{G}_{2}$. This is because the assumption that the fading is constant over the two consecutive transmission instants is no longer valid in this situation. Here, the two consecutive transmission instants are associated with two adjacent subcarriers in the OFDM symbol, and the fading variation is relatively fast in the frequency domain. Therefore, the orthogonality of the space-time code has been destroyed by the frequency-domain variation of the fading envelope. At the receiver, the combiner can no longer separate the two different transmitted signals $x_{1}$ and $x_{2}$. Specifically, the signals interfere with each other. The increase in SNR does not improve the performance of the STB code $\mathbf{G}_{2}$, since this also increases the power of the interfering signal. We will address this issue more explicitly in Section III-B. By contrast, the $\mathrm{TC}(2,1,3)$ channel codec succeeds in overcoming this problem. However, we will show in Section III-B that the concatenated

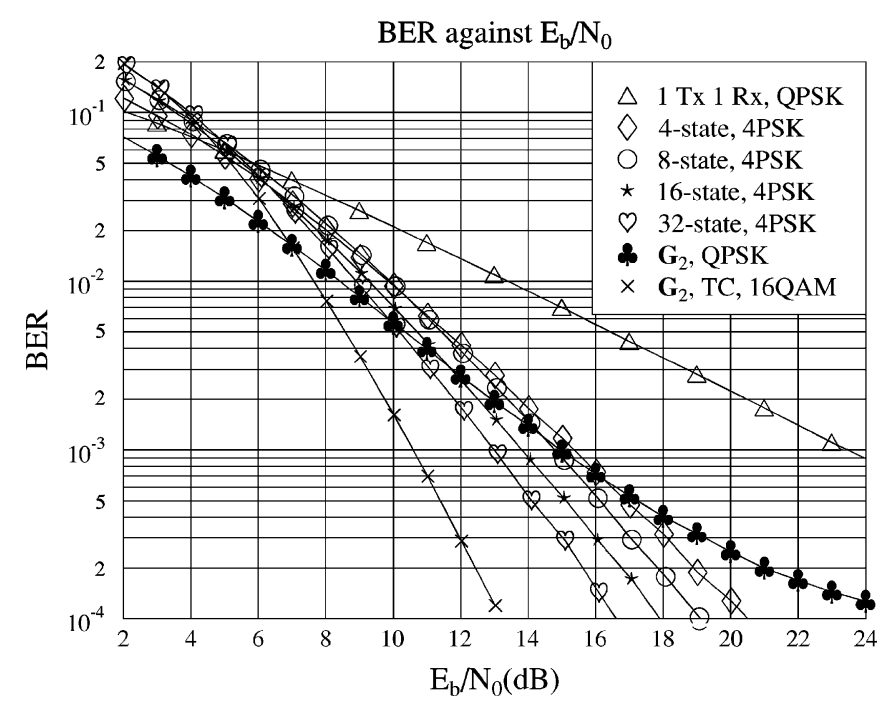

Fig. 5. BER performance comparison between various 4PSK STT codes and the STB code $\mathbf{G}_{2}$ concatenated with the TC $(2,1,3)$ code using one receiver and the 128-subcarrier OFDM modem over a channel having a CIR characterized by two equal-power rays separated by a delay spread of $5 \mu \mathrm{s}$. The maximum Doppler frequency was $200 \mathrm{~Hz}$. The effective throughput was $2 \mathrm{BPS}$, and the coding parameters are shown in Tables I-III.

channel-coded scheme exhibits the same residual BER problem if the channel's variation becomes more rapid.

In Fig. 5, we provide the corresponding BER performance comparison between the 4PSK STT codes and the STB code $\mathbf{G}_{2}$ concatenated with the $\mathrm{TC}(2,1,3)$ code using one receiver and the 128-subcarrier OFDM modem over a channel characterized by two equal power rays separated by a delay spread of $5 \mu \mathrm{s}$ and having a maximum Doppler frequency of $200 \mathrm{~Hz}$. Again, we show in the figure that the 2 BPS throughput concatenated $\mathbf{G}_{2} / \mathrm{TC}(2,1,3)$ scheme outperforms the 2 BPS STT codes using no channel coding. At a BER of $10^{-4}$, the concatenated channel coded scheme is at least 2-dB superior in SNR terms to the STT codes using no channel codes. At high $E_{b} / N_{0}$ values, the STB code $\mathbf{G}_{2}$ again exhibits a residual BER. On the other hand, at low $E_{b} / N_{0}$ values, the latter outperforms the concatenated $\mathbf{G}_{2} / \mathrm{TC}(2,1,3)$ channel-coded scheme as well as the STT codes using no channel coding.

In [5] and [24] and in Section II-C, we have derived the complexity estimates of the TC decoders and STT decoders, respectively. By employing (2) and the equations in [24], we compare the performance of the schemes studied while considering their approximate complexity. Our performance comparison of the various schemes was carried out on the basis of the coding gain defined as the $E_{b} / N_{0}$ difference, expressed in decibels, at FER $=10^{-3}$ between the schemes studied and the uncoded single-transmitter, single-receiver system having the same throughput of 2 BPS. In Fig. 6, we show our coding gain versus estimated complexity comparison for the various 4PSK STT codes and the STB code $\mathbf{G}_{2}$ concatenated with the $\mathrm{TC}(2,1,3)$ code using one as well as two receivers. The 128-subcarrier OFDM modem transmitted over the channel having a CIR of two equal power rays separated by a delay spread of $5 \mu \mathrm{s}$ and a maximum Doppler frequency of $200 \mathrm{~Hz}$. The estimated complexity of the STT codes was increased by increasing 


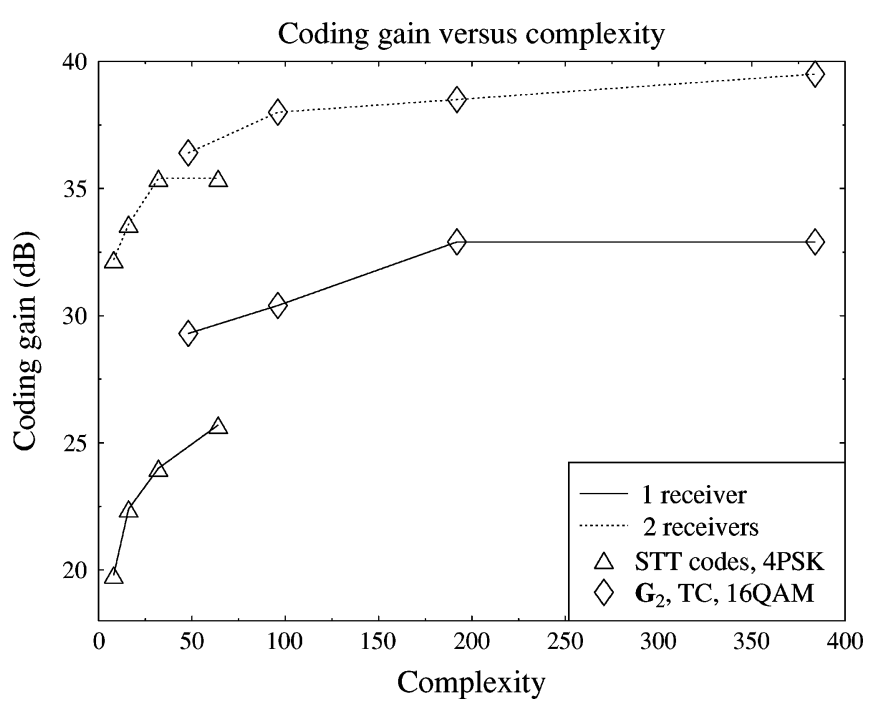

Fig. 6. Coding gain versus estimated complexity for the various 4PSK STT codes and the STB code $\mathbf{G}_{2}$ concatenated with the $\mathrm{TC}(2,1,3)$ code using one as well as two receivers and the 128-subcarrier OFDM modem over a channel having a CIR characterized by two equal-power rays separated by a delay spread of $5 \mu \mathrm{s}$. The maximum Doppler frequency was $200 \mathrm{~Hz}$. The effective throughput was 2 BPS, and the coding parameters are shown in Tables I-III.

the number of trellis states. By constrast, the estimated complexity of the $\mathrm{TC}(2,1,3)$ code was increased by increasing the number of turbo iterations. The coding gain of the concatenated $\mathbf{G}_{2} / \mathrm{TC}(2,1,3)$ scheme using one, two, four, and eight iterations is shown in Fig. 6. It can be seen that the concatenated scheme outperforms the space-time trellis codes using no channel coding, even though the number of turbo iterations was only one. Moreover, the improvement in coding gain was obtained at an estimated complexity comparable to that of the 32-state 4PSK space-time trellis code using no channel coding. From the figure, we can also see that the performance gain of the concatenated $\mathbf{G}_{2} / \mathrm{TC}(2,1,3)$ channel-coded scheme over the STT codes becomes lower when the number of receivers is increased to two.

Due to space limitations, the FER and BER curves of the corresponding 8PSK-based 3-BPS transceivers are not included here. However, similarly to the 2-BPS schemes of Fig. 6, we compare the performance of the 3-BPS throughput schemes by considering their approximate decoding complexity. The derivation of the estimated complexity has been detailed in [5] and [24] and Section II-C. As mentioned previously, the performance comparison of the various schemes was made on the basis of the coding gain defined as the $E_{b} / N_{0}$ difference, expressed in decibels, at a FER $=10^{-3}$ between the schemes investigated and the uncoded single-transmitter, single-receiver system having a throughput of 3 BPS. In Fig. 7, we show the associated coding gain versus estimated complexity curves for the 8PSK spacetime trellis codes using no channel coding and the STB code $\mathbf{G}_{2}$ concatenated with the $\mathrm{TC}(2,1,3)$ code using one and two receivers and the 128-subcarrier OFDM modem. For the sake of consistency, the CIR again exhibited two equal power rays separated by a delay spread of $5 \mu$ s and a maximum Doppler frequency of $200 \mathrm{~Hz}$. Again, the estimated complexity of the STT codes was increased by increasing the number of states. On the other hand, the estimated complexity of the $\mathrm{TC}(2,1,3)$ code

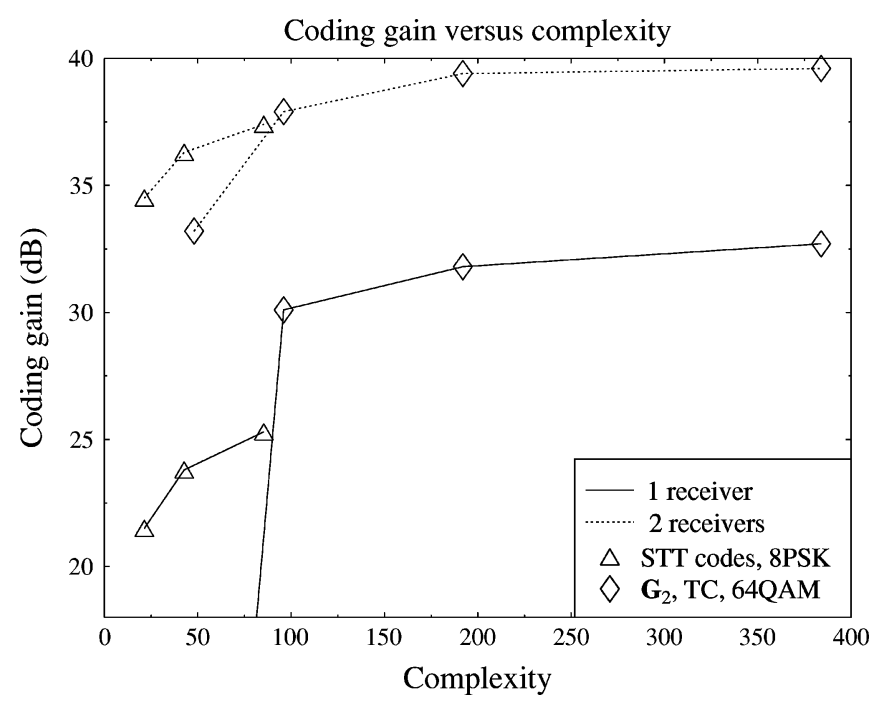

Fig. 7. Coding gain versus estimated complexity for the various 8PSK STT codes and the STB code $\mathbf{G}_{2}$ concatenated with the TC $(2,1,3)$ code using one and two receivers and the 128 -subcarrier OFDM modem over a channel having a CIR characterized by two equal-power rays separated by a delay spread of $5 \mu \mathrm{s}$. The maximum Doppler frequency was $200 \mathrm{~Hz}$. The effective throughput was 3 BPS, and the coding parameters are shown in Tables I-III.

was increased by increasing the number of iterations. The coding gain of the concatenated channel-coded scheme invoking one, two, four, and eight iterations is shown in Fig. 7. Previously, in Fig. 6, we have shown that the concatenated $\mathrm{TC}(2,1,3)$-coded scheme using one iteration outperformed the STT codes using no channel coding. However, in Fig. 7, the concatenated scheme does not exhibit the same performance trend. For the case of one receiver, the concatenated scheme using one iteration has a negative coding gain and exhibits a saturation effect. This is again due to the employment of the high-order 64QAM scheme, which has a preponderance to exceed the channel's capacity. Again, we can also see that the performance gain of the concatenated $\mathbf{G}_{2} / \mathrm{TC}(2,1,3)$-coded scheme over the STT codes using no channel coding becomes smaller when the number of receivers is increased to two. Having studied the performance of the various schemes over the channel characterized by the two-path, $5-\mu$ dispersion CIR at a fixed Doppler frequency of $200 \mathrm{~Hz}$, we next study the effects of varying the Doppler frequency.

\section{B. Effect of Delay Spreads}

In this section, we will study how the variation of the delay spread between the two paths of the channel affects system performance. By varying the delay spread, the channel's frequency-domain response varies as well. In Fig. 8, we show the fading amplitude variation of the 128 subcarriers in an OFDM symbol for a delay spread of (a) 5, (b) 10, (c) 20, and (d) $40 \mu \mathrm{s}$. It can be seen from the figure that the fading amplitudes vary more rapidly when the delay spread is increased. For the code STB $\mathbf{G}_{2}$, the fading envelopes of the two consecutive transmission instants of antennas $T x 1$ and $T x 2$ are assumed to be constant [1]. In Fig. 8(d), we see that the variation of the frequency-domain fading amplitudes is so dramatic that we can no longer assume that the fading envelopes are constant for two consecutive transmission instants. The variation of the 


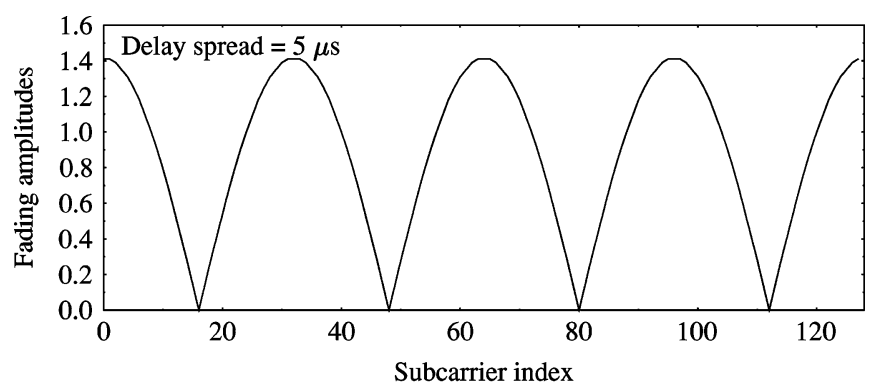

(a)

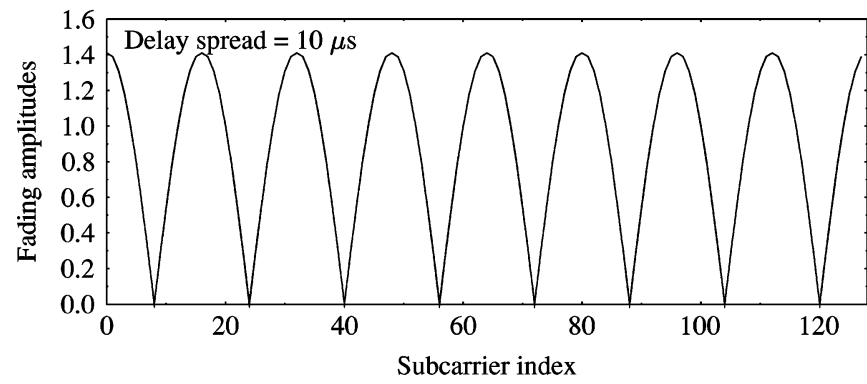

(b)

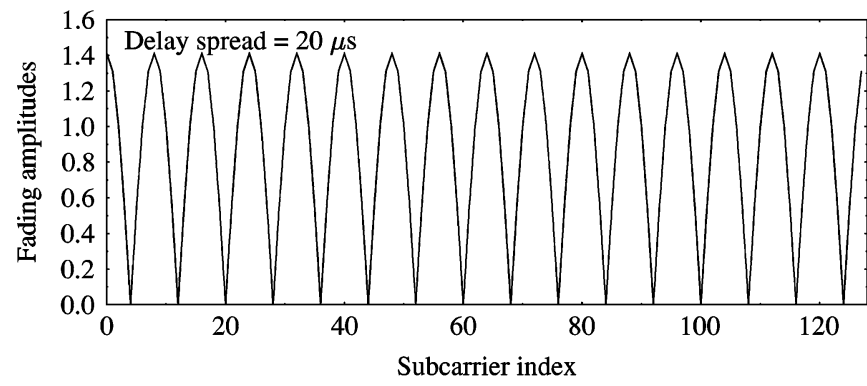

(c)

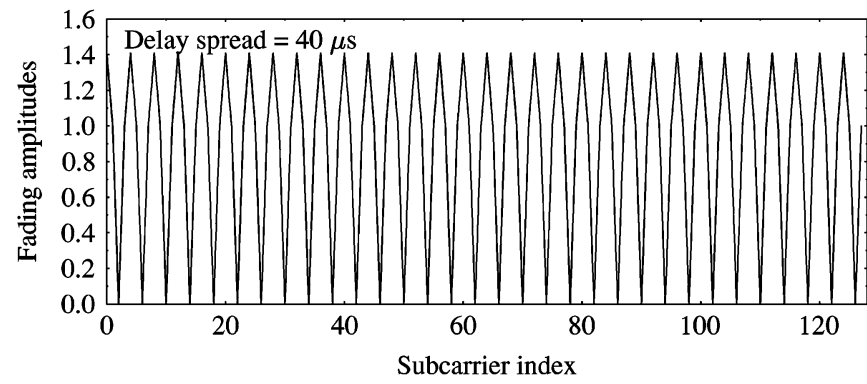

(d)

Fig. 8. Frequency-domain fading magnitudes of the 128 subcarriers in an OFDM symbol for an equal tap gain two-path channel having a tap separation of (a) 5, (b) 10 , (d) 20 , and (c) $40 \mu \mathrm{s}$.

frequency-domain fading envelope will eventually destroy the orthogonality of the STB code $\mathbf{G}_{2}$.

When communicating over dispersive channels, the two transmission instants of the $\mathbf{G}_{2}$ code may no longer be assumed to be associated with the same complex frequency-domain channel transfer function values. The figure shows the baseband representation of the simple twin-transmitter space-time block code $\mathbf{G}_{2}$ of (1) using one receiver. At the receiver, we have

$$
\begin{aligned}
& y_{1}=h_{1,1} x_{1}+h_{2,1} x_{2}+n_{1} \\
& y_{2}=-h_{1,2} \bar{x}_{2}+h_{2,2} \bar{x}_{1}+n_{2}
\end{aligned}
$$

where $y_{1}$ is the first received signal, and $y_{2}$ is the second. Both signals $y_{1}$ and $y_{2}$ are passed to the combiner in order to extract the signals $x_{1}$ and $x_{2}$. Aided by the channel estimator, which in this example provides perfect estimation of the diversity channel's frequency-domain transfer functions, the combiner performs simple signal processing in order to separate the signals $x_{1}$ and $x_{2}$. Specifically, in order to extract the signal $x_{1}$, it combines signals $y_{1}$ and $y_{2}$ as follows:

$$
\begin{aligned}
\tilde{x}_{1}= & \bar{h}_{1,1} y_{1}+h_{2,2} \bar{y}_{2} \\
= & \bar{h}_{1,1} h_{1,1} x_{1}+\bar{h}_{1,1} h_{2,1} x_{2}+\bar{h}_{1,1} n_{1}-h_{2,2} \bar{h}_{1,2} x_{2} \\
& +h_{2,2} \bar{h}_{2,2} x_{1}+h_{2,2} \bar{n}_{2} \\
= & \left(\left|h_{1,1}\right|^{2}+\left|h_{2,2}\right|^{2}\right) x_{1}+\left(\bar{h}_{1,1} h_{2,1}-h_{2,2} \bar{h}_{1,2}\right) x_{2} \\
& +\bar{h}_{1,1} n_{1}+h_{2,2} \bar{n}_{2} .
\end{aligned}
$$

A similarly simple symmetric expression may be generated for $x_{2}$. In contrast to the prefect cancellation scenario of [1], we can see from (5) that the signals $x_{1}$ and $x_{2}$ now interfere with each other since the second round-bracketed term becomes nonzero. Hence, we can no longer cancel the cross-coupling of signals $x_{2}$ and $x_{1}$ in (5) unless the fading envelopes satisfy the condition of $h_{1,1}=h_{1,2}$ and $h_{2,1}=h_{2,2}$.

At high SNRs, the noise power is insignificant compared to the transmitted power of the signals $x_{1}$ and $x_{2}$. Therefore, we can ignore the noise terms $n$ in (5) However, the interference signal's power increases as we increase the transmission power. Assuming that both the signals $x_{1}$ and $x_{2}$ have an equivalent signal power, we can then express the signal-to-interference ratio (SIR) for signal $x_{1}$ as

$$
\operatorname{SIR}=\frac{\left|h_{1,1}\right|^{2}+\left|h_{2,2}\right|^{2}}{\bar{h}_{1,1} h_{2,1}-h_{2,2} \bar{h}_{1,2}}
$$

and again, a similarly simple symmetric expression may be generated for the SIR of $x_{2}$.

In Fig. 9, we show the FER performance of the STB code $\mathbf{G}_{2}$ concatenated with the $\mathrm{TC}(2,1,3)$ code using one receiver and the 128-subcarrier 16QAM OFDM modem as a function of the delay spread. The CIR has two equal power rays separated by various delay spreads and a maximum Doppler frequency of $200 \mathrm{~Hz}$. As we can see in (6), we have SIR $\rightarrow \infty$ if $h_{1,1}=h_{1,2}$ and $h_{2,1}=h_{2,2}$. On the other hand, we encounter SIR $\rightarrow 1$ if $h_{1,1}=\delta_{1} h_{1,2}$ and $h_{2,1}=\delta 2 h_{2,2}$, where $\delta_{1} \rightarrow \infty$, and $\delta_{2} \rightarrow \infty$. Since the SIR decreases, when the delay spread increases due to the rapidly fluctuating frequency-domain fading envelopes, as shown in Fig. 8, we can see in Fig. 9 that the performance of the concatenated scheme degrades when increasing the delay spread. When the delay spread is more than $15 \mu \mathrm{s}$, we can see from the figure that the concatenated scheme exhibits the previously observed BER-curve flattening effect. Furthermore, the error floor of the concatenated scheme becomes higher as the delay spread is increased.

In Fig. 10, we quantify the $E_{b} / N_{0}$ value required for maintaining FER $=10^{-3}$ versus the delay spread for the 32-state 4PSK STT code and for the STB code $\mathbf{G}_{2}$ concatenated with 


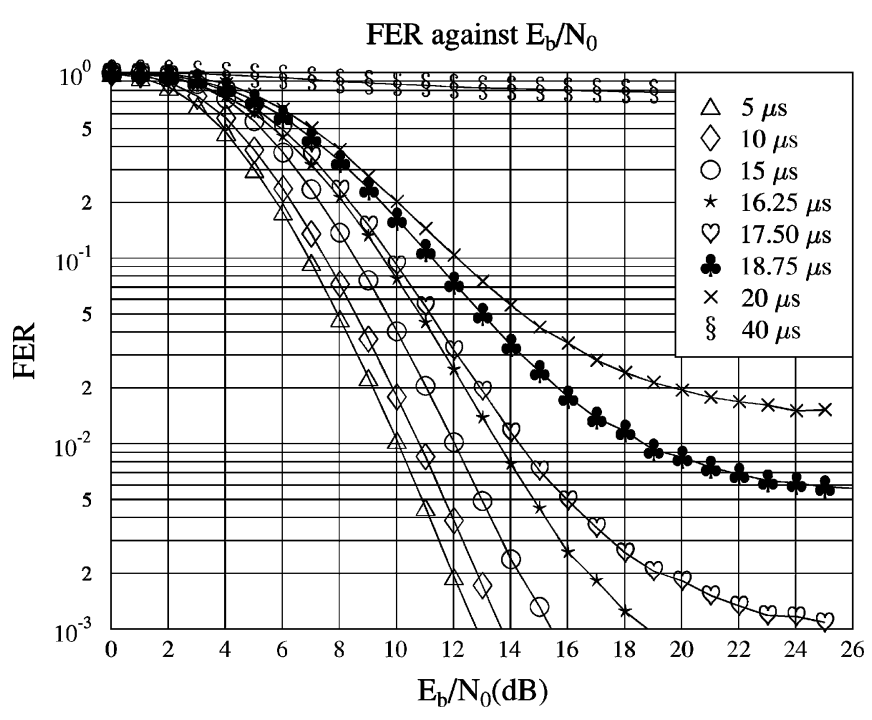

Fig. 9. FER performance of the STB code $\mathbf{G}_{2}$ concatenated with the TC $(2,1,3)$ code using one receiver, the 128 -subcarrier OFDM modem, and 16QAM. The CIR exhibits two equal-power rays separated by various delay spreads and a maximum Doppler frequency of $200 \mathrm{~Hz}$. The coding parameters are shown in Tables I-III.

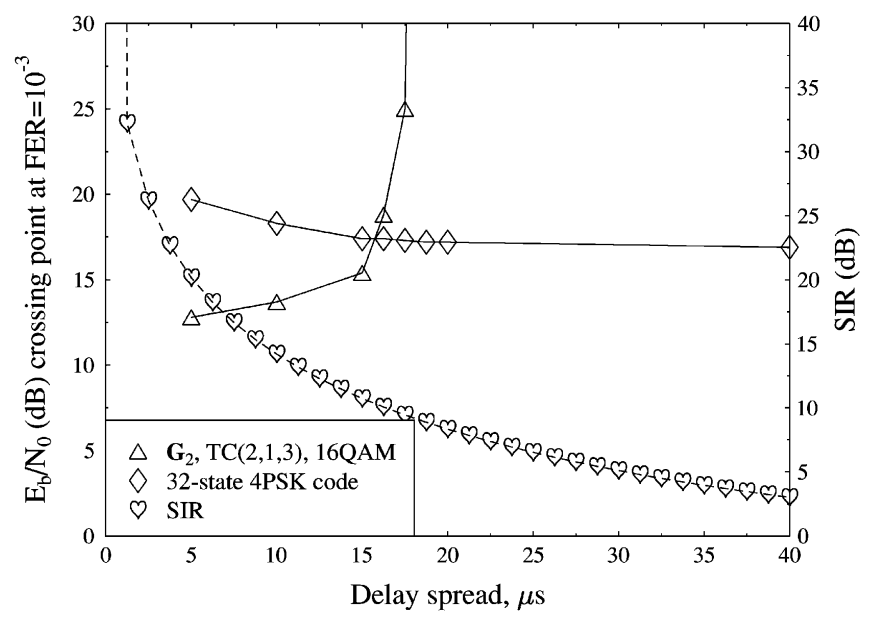

Fig. 10. $E_{b} / N_{0}$ values required for maintaining FER $=10^{-3}$ versus delay spreads for the 32-state 4PSK space-time trellis code and for the space-time block code $\mathbf{G}_{2}$ concatenated with the $\mathrm{TC}(2,1,3)$ code using one receiver and the 128-subcarrier OFDM modem. The CIR exhibited two equal power rays separated by various delay spreads and a maximum Doppler frequency of $200 \mathrm{~Hz}$. The effective throughput was 2 BPS, and the coding parameters are shown in Tables I-III. The SIR of various delay spreads are shown as well.

the $\operatorname{TC}(2,1,3)$ code using one receiver and the 128 -subcarrier OFDM modem. The CIR exhibited two equal power rays separated by various delay spreads. The maximum Doppler frequency was $200 \mathrm{~Hz}$. We can see in the figure that the performance of the 32-state 4PSK space-time trellis code does not vary significantly with the delay spread. However, the concatenated TC $(2,1,3)$-coded scheme suffers severe performance degradation upon increasing the delay spread, as evidenced by the associated error floors shown in Fig. 9. The SIR associated with the various delay spreads was obtained using computer simulations, and the associated SIR values are also shown in Fig. 10, denoted by the hearts. As expected, the calculated SIR decreases with the delay spread. We can see in the figure that

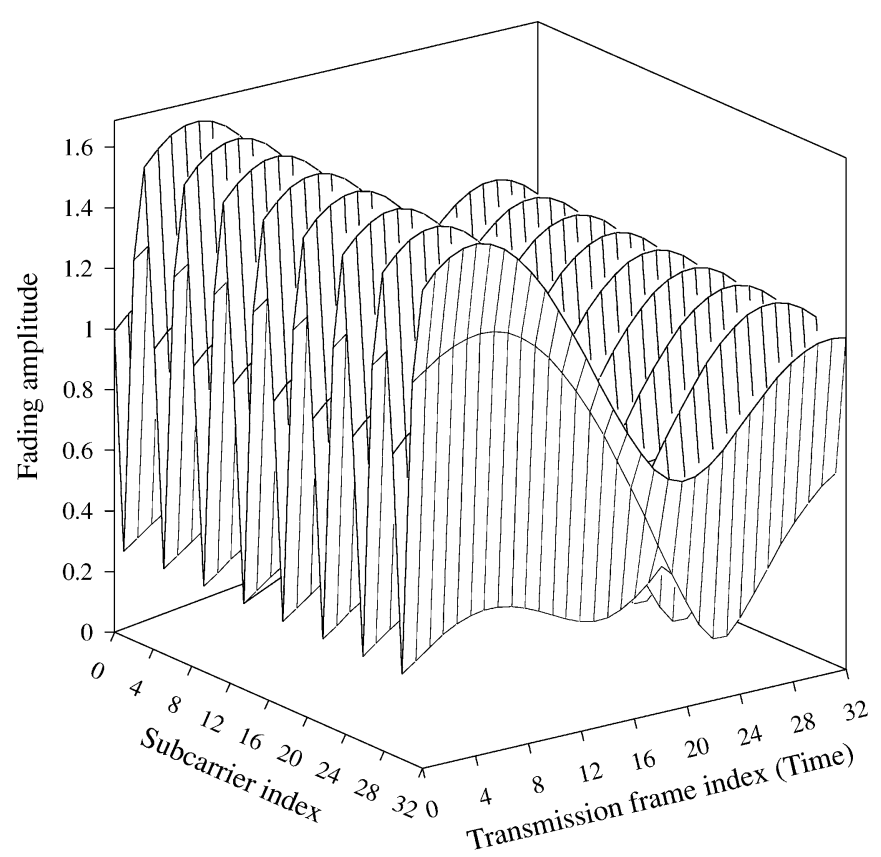

Fig. 11. Fading amplitude versus time and frequency for various 128subcarrier OFDM symbols over the two-path channel exhibiting two equal power rays separated by a delay spread of $40 \mu$ s and maximum Doppler frequency of $100 \mathrm{~Hz}$.

the performance of the concatenated $\mathbf{G}_{2} / \mathrm{TC}(2,1,3)$ scheme suffers severe degradation when the delay spread is in excess of $15 \mu \mathrm{s}$, as indicated by the near-vertical curve marked by triangles. If we relate this curve to the SIR curve marked by the hearts, we can see from the figure that the SIR is approximately $10 \mathrm{~dB}$. Hence, the SIR of the concatenated $\mathbf{G}_{2} / \mathrm{TC}(2,1,3)$ scheme has to be more than $10 \mathrm{~dB}$ for it to outperform the STT codes using no channel coding.

\section{High-Delay System}

Previously, we have provided simulation results for a lowdelay OFDM symbol-by-symbol decoded system. More explicitly, the received OFDM symbol had to be demodulated and decoded on an OFDM symbol-by-symbol basis in order to provide decoded bits for example for a low-delay interactive speech [33] or video [34] source decoder. Therefore, the two transmission instants of the STB code $\mathbf{G}_{2}$ had to be in the same OFDM symbol. Hence, as argued previously, they were allocated to the adjacent OFDM subcarriers in our preceding studies. Moreover, we have shown in Fig. 8 that the variation of the frequency-domain fading amplitudes along the subcarriers becomes more severe as we increase the delay spread of the two rays. In Fig. 11, we show both the frequency-domain and time-domain fading amplitudes of the channels' fading amplitudes for a fraction of the subcarriers in the 128-subcarrier OFDM symbols over the previously used two-path channel having two equal power rays separated by a delay spread of $40 \mu \mathrm{s}$. The maximum Doppler frequency was set here to $100 \mathrm{~Hz}$. It can be clearly seen from the figure that the fading amplitude variation versus time is slower than that versus the subcarrier index within the OFDM symbols. This 


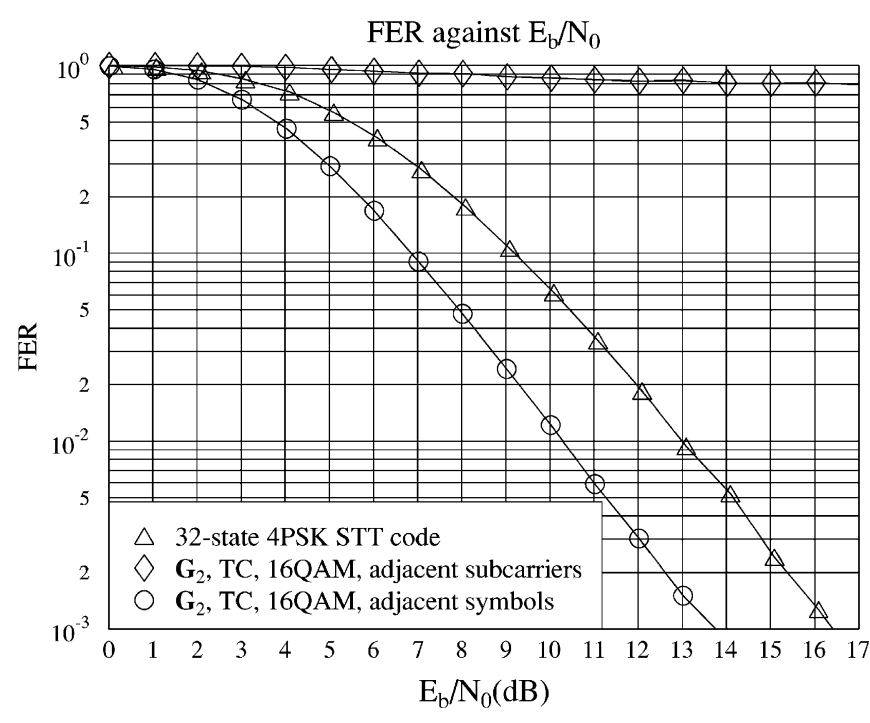

Fig. 12. FER performance comparison between adjacent subcarriers and adjacent OFDM symbols allocation for the STB code $\mathbf{G}_{2}$ concatenated with the $\mathrm{TC}(2,1,3)$ code using one receiver, the 128 -subcarrier OFDM modem, and 16QAM over a channel having a CIR characterized by two equal-power rays separated by a delay spread of $40 \mu \mathrm{s}$. The maximum Doppler frequency was $100 \mathrm{~Hz}$. The coding parameters are shown in Tables I-III.

implies that the SIR attained would be higher if we were to allocate the two transmission instants of the STB code $\mathbf{G}_{2}$ to the same subcarrier of consecutive OFDM symbols. This increase in SIR is achieved by doubling the delay of the system, since in this scenario, two consecutive OFDM symbols have to be decoded before all the received data becomes available.

In Fig. 12, we show our FER performance comparison for the preceding two scenarios, namely, using two adjacent subcarriers and the same subcarrier in two consecutive OFDM symbols for the STB code $\mathbf{G}_{2}$ concatenated with the $\mathrm{TC}(2,1,3)$ code using one 128-subcarrier 16QAM OFDM receiver. As before, the CIR exhibited two equal power rays separated by a delay spread of $40 \mu \mathrm{s}$ and a maximum Doppler frequency of $100 \mathrm{~Hz}$. It can be seen from the figure that there is a severe performance degradation if the two transmission instants of the STB $\mathbf{G}_{2}$ are allocated to two adjacent subcarriers. This is evidenced by the near-horizontal curve marked by diamonds across the figure. On the other hand, upon assuming that having a delay of two OFDM-symbol durations does not pose any problems in terms of real-time interactive communications, we can allocate the two transmission instants of the space-time block code $\mathbf{G}_{2}$ to the same subcarrier of two consecutive OFDM symbols. From Fig. 12, we can observe a dramatic improvement over the previous allocation method. Furthermore, the figure also indicates that by tolerating a two OFDM-symbol delay, the concatenated $\mathbf{G}_{2} / \mathrm{TC}(2,1,3)$ scheme outperforms the 32-state 4PSK space-time trellis code by approximately $2 \mathrm{~dB}$ in terms of the required $E_{b} / N_{0}$ value at a FER of $10^{-3}$.

Since the two transmission instants of the STB code $\mathbf{G}_{2}$ are allocated to the same subcarrier of two consecutive OFDM symbols, it is the maximum Doppler frequency that would affect the performance of the concatenated scheme more gravely, rather than the delay spread. Hence, we extended our studies to con-

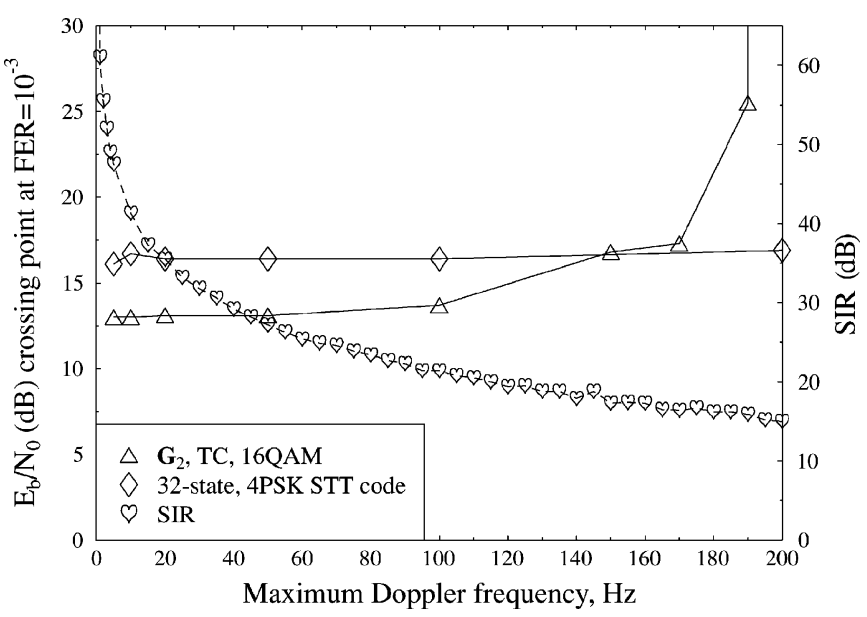

Fig. 13. $E_{b} / N_{0}$ value required for maintaining FER $=10^{-3}$ versus the $\max$ imum Doppler frequency for the 32-state 4PSK STT code and for the adjacent OFDM symbols allocation of the STB code $\mathbf{G}_{2}$ concatenated with the $\mathrm{TC}(2,1,3)$ code using one receiver and the 128 -subcarrier OFDM modem. The CIR exhibited two equal power rays separated by a delay spread of $40 \mu \mathrm{s}$. The effective throughput was 2 BPS, and the coding parameters are shown in Tables I-III. The SIR of various maximum Doppler frequencies are shown as well.

sider the effects of the maximum Doppler frequency on the performance of the concatenated $\mathbf{G}_{2} / \mathrm{TC}(2,1,3)$ scheme. Specifically, Fig. 13 shows the $E_{b} / N_{0}$ values required for maintaining FER $=10^{-3}$ versus the Doppler frequency for the 32-state 4PSK STT code and for the STB code $\mathbf{G}_{2}$ concatenated with the $\mathrm{TC}(2,1,3)$ code using one 128-subcarrier 16QAM OFDM receiver when mapping the two transmission instants to the same subcarrier of two consecutive OFDM symbols. The channel exhibited two equal power rays separated by a delay spread of $40 \mu \mathrm{s}$ and various maximum Doppler frequencies. The SIR achievable at various maximum Doppler frequencies is also shown in Fig. 13. Again, we can see that the performance of the concatenated $\mathbf{G}_{2} / \mathrm{TC}(2,1,3)$ scheme suffers severely if the maximum Doppler frequency is above $160 \mathrm{~Hz}$. More precisely, we can surmise that in order for the concatenated scheme to outperform the 32-state 4PSK space-time trellis code, the SIR should be at least $15 \mathrm{~dB}$, which is about the same as the required SIR in Fig. 10. From Figs. 10 and 13, we can conclude that the concatenated $\mathbf{G}_{2} / \mathrm{TC}(2,1,3)$ scheme performs better if the SIR is in excess of about $10-15 \mathrm{~dB}$.

\section{SPACE-Time Coded AdAPtive Modulation for OFDM}

\section{A. Adaptive Modulation}

Adaptive modulation was proposed by Steele and Webb [35], [36] in order to combat the time-variant fading of mobile channels. The main idea of adaptive modulation [37] is that when the channel quality is favorable, higher order modulation modes are employed in order to increase the throughput of the system. On the other hand, more robust but lower throughput modulation modes are employed if the channel quality is low. This simple but elegant idea has motivated a number of researchers to probe further [16], [17], [37]-[45]. The success of this approach led to its 
employment in the third-generation wireless system's [46], [47] high-speed data packet access mode.

Recently adaptive modulation was also invoked in the context of OFDM, which was termed adaptive OFDM (AOFDM) [16], [17], [41], [42], [48]. AOFDM exploits the variation of the signal quality, both in the time domain as well as in the frequency domain. In what is known as subband adaptive OFDM transmission, all subcarriers in an AOFDM symbol are split into blocks of adjacent subcarriers referred to as subbands. The same modulation scheme is employed for all subcarriers of the same subband. This substantially simplifies the task of signaling the modulation modes, since there are typically four modes and, for example, 32 subbands, requiring a total of 64 AOFDM mode signaling bits.

\section{B. Turbo-Coded and Space-Time-Coded Adaptive OFDM}

In this section, the adaptive OFDM philosophy portrayed, for example, in [16], [17], [41], [42], and [49] is extended in order to exploit the advantages of multiple transmit and receive antennas. Additionally, turbo coding is employed for improving the performance of the system. The system schematic of the turbo-coded and space-time-coded adaptive OFDM system is identical to that in Fig. 1, except that, additionally, the AOFDM modes have to be signaled from the receiver estimating the channel quality to the remote transmitter using this information for activating the required AOFDM mode. Similarly to Fig. 1, random data bits are generated and encoded by the $\operatorname{TC}(2,1,3)$ encoder using an octal generator polynomial of $(7,5)$. Various coding rates were used in conjunction with the different modulation mode, which were generated by appropriately puncturing [5] the $\mathrm{TC}(2,1,3)$ codec. The encoded bits were channel interleaved and passed to the modulator. The choice of the modulation scheme to be used by the transmitter for its next OFDM symbol is determined by the channel quality estimate of the receiver based on the current OFDM symbol. In this study, we assumed perfect channel quality estimation and perfect signaling of the required modem mode of each subband based on the channel quality estimate acquired during the current OFDM symbol. Aided by the perfect channel quality estimator, the receiver determines the highest throughput modulation mode to be employed by the transmiter for its next transmission while maintaining the system's target BER. Five possible transmission modes were employed in our investigations, which are no transmission (NoTx), BPSK, QPSK, 16QAM, and 64QAM. In order to simplify the task of signaling the required modulation modes, we employed the subband adaptive OFDM transmission scheme detailed, for example, in [16], [17], [41], [42], and [49]. The modulated signals were then passed to the encoder of the STB code $\mathbf{G}_{2}$. The space-time encoded signals were OFDM modulated and transmitted by the corresponding antennas.

The number of receivers invoked constitutes a design parameter. The received signals were OFDM demodulated and passed to the space-time decoders. Log-MAP [29] decoding of the received space-time signals was performed in order to provide soft-outputs for the $\operatorname{TC}(2,1,3)$ decoder. Assuming that the demodulator of the receiver has perfect knowledge of the instan-

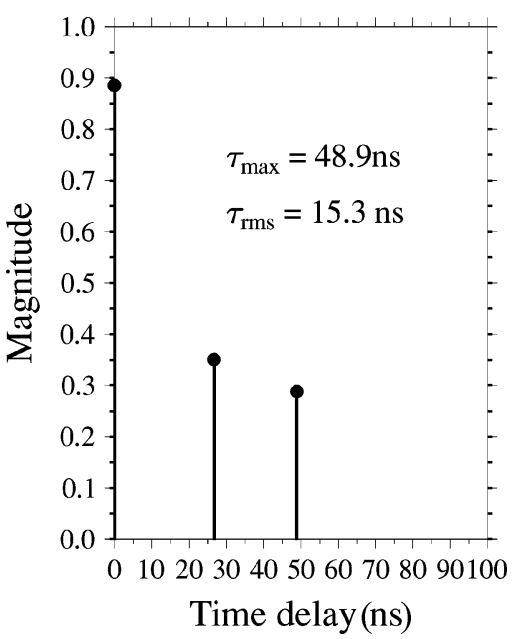

Fig. 14. Short WATM channel impulse response.

taneous channel quality, this information is passed to the transmitter in order to determine its next AOFDM modulation mode allocation. The received bits were then channel deinterleaved and passed to the TC decoder, which employs the Log-MAP decoding algorithm [28]. The decoded bits were finally passed to the sink for calculation of the BER.

\section{Simulation Results}

1) Shortened WATM Channel: The shortened WATM channel was used, and its CIR profile is portrayed in Fig. 14. The system used 512 subcarriers, and each OFDM symbol was extended with a cyclic prefix of length 64 . The sampling rate was $225 \mathrm{Msamples} / \mathrm{s}$, and the carrier frequency was $60 \mathrm{GHz}$. In [16] and [17] two WATM CIRs were used, namely, a five-path and a three-path model, where the latter was referred to as the shortened WATM CIR. This CIR was used also in our investigations here, which is depicted in Fig. 14, where the longest delay path arrived at a delay of $48.9 \mathrm{~ns}$, which corresponds to 11 sample periods at the 225 Msamples/s sampling rate.

2) Space-Time Coded Adaptive OFDM: In this section, we employ the fixed threshold-based modem mode selection algorithm, which was also used in [16] and [17], adapting the techniques of [39], [50], and [51] for serial modems. It was assumed that the channel quality was constant for all the symbols in a transmission burst, i.e., that the channel's fading envelope varied slowly across the transmission burst. Under these conditions, all the transmitted symbols are modulated using the same modulation mode, chosen according to the predicted SNR. Torrance optimized the modem mode switching thresholds [39], [50], [51] for the target BERs of $10^{-2}$ and $10^{-4}$, which will be appropiate for a high-BER speech system and for a low-BER data system, respectively. The resulting SNR switching thresholds for activating a given modulation mode in a slowly Rayleigh fading, narrowband channel are given in Table VI for both systems. Assuming perfect channel quality estimation, the instantaneous channel SNR is measured by the receiver, and the information is passed to the modulation mode selection switch at the transmitter using the system's control channel. This side-information signaling does not constitute a 
TABLE VI

OPTIMIZED SWITCHING LEVELS QUOTED FROM [39] FOR ADAPTIVE MODUlation OVER RAYLEIGH FADING CHANNELS FOR THE SPEECH AND DATA SySTEMS, SHOWN IN INSTANTANEOUS CHANNEL SNR (IN DECIBELS)

\begin{tabular}{|c|c|c|c|c|c|}
\hline System & NoTx & BPSK & QPSK & 16QAM & 64QAM \\
\hline Speech & $-\infty$ & 3.31 & 6.48 & 11.61 & 17.64 \\
\hline Data & $-\infty$ & 7.98 & 10.42 & 16.76 & 26.33 \\
\hline
\end{tabular}

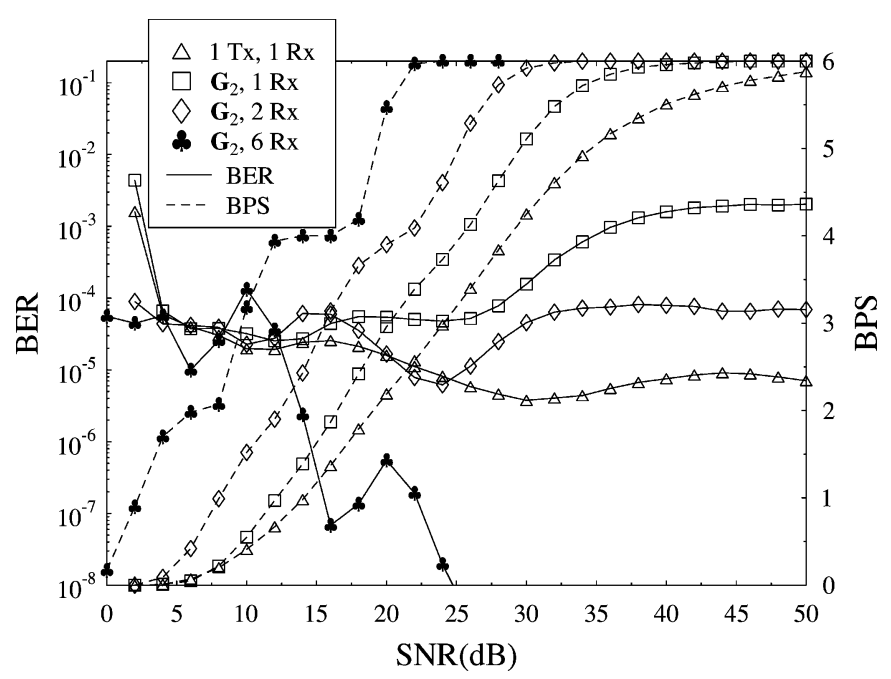

Fig. 15. BER and BPS performance of 16-subband AOFDM employing the STB code $\mathbf{G}_{2}$ using multiple receivers for a target BER of $10^{-4}$ over the shortened WATM channel shown in Fig. 14. The switching thresholds are shown in Table VI.

problem, since state-of-the-art wireless systems, such as, for example, IMT-2000 [25], have a high-rate, low-delay signaling channel. This modem mode signaling feedback information is utilized by the transmitter for selecting the next modulation mode. Specifically, a given modulation mode is selected if the instantaneous channel SNR perceived by the receiver exceeds the corresponding switching levels shown in Fig. 15, depending on the target BER.

As mentioned previously, the adaptation algorithm of [39], [50] and [51] assumes constant instantaneous channel SNR over the whole transmission burst. However, in the case of an OFDM system transmitting over frequency-selective channels, the channels' quality varies across the different subcarriers. In [16], [17], and [49], the lowest quality subcarrier of the subband is employed for controlling the adaptation algorithm, which is based on the switching thresholds given in Table VI. Again, this approach significantly simplifies the signaling, and therefore, it was also adopted in our investigations.

In Fig. 15, we show the BER and BPS performance of the 16subband AOFDM scheme employing the STB code $\mathbf{G}_{2}$ in conjunction with multiple receivers and a target BER of $10^{-4}$ over the shortened WATM channel shown in Fig. 14. The switching thresholds are shown in Table VI. The performance of the conventional AOFDM scheme using no diversity [16], [17] is also shown in the figure. From Fig. 15, we can see that the BPS performance of the space-time coded AOFDM scheme using one receiver is better than that of the conventional AOFDM scheme.
The associated performance gain improves as the throughput increases. At a throughput of 6 BPS, the space-time coded scheme outperforms the conventional scheme by at least $10 \mathrm{~dB}$ in $E_{b} / N_{0}$ terms. However, we notice in Fig. 15 that as a secondary effect, the BER performance of the space-time coded AOFDM scheme using one receiver degrades as we increase the average channel SNR. Again, this problem is due to the interference of signals $x_{1}$ and $x_{2}$ caused by the rapidly varying frequency-domain fading envelope across the subcarriers. We note that it is possible to take into account this interference source in the calculation of the effective signal to interference plus noise ratio (SINR).

At high SNRs, predominantly 64QAM was employed. Since the constellation points in 64QAM are densely packed, this modulation mode is more sensitive to the "cross-talk" of the signals $x_{1}$ and $x_{2}$. This limited the BER performance to $10^{-3}$ even at high SNRs. However, at SNRs lower than $30 \mathrm{~dB}$, typically more robust modulation modes were employed, and hence, the target BER of $10^{-4}$ was readily met. We will show in the next section that this problem can be overcome by employing turbo channel coding in the system.

In Fig. 15, we also observe that the BER and BPS performance improves as we increase the number of AOFDM receivers, since the dispersion-induced interference between the signals $x_{1}$ and $x_{2}$ is eliminated. With six AOFDM receivers, the BER of the system drops below $10^{-8}$ when the average channel SNR exceeds $25 \mathrm{~dB}$, and in the SNR-range investigated, there was no sign of a residual BER. It is also interesting to note the undulating nature of the BER curve, which is due to the fact that fixed AOFDM mode switching thresholds were used, and when activating switching to a higher throughput AOFDM mode, the BER inevitably increased first, before decreasing upon further increasing of the SNR. It is also worth mentioning that the AOFDM switching thresholds were optimized following the AOFDM design procedures of [16] and [17]. However, more recent research augmented the fact the optimum switching thresholds are slightly SNR-dependent [17], [37], and hence, the thresholds employed here were somewhat suboptimum.

In Fig. 16, we show the instantaneous channel SNR experienced by the 512-subcarrier OFDM symbols for a single-transmitter, single-receiver scheme and for the STB code $\mathrm{G}_{2}$ using two receivers over the shortened WATM channel. The average channel SNR is $10 \mathrm{~dB}$. We can see in Fig. 16 that the variation of the instantaneous channel SNR for a single transmitter and single receiver is fast and severe. The instantaneous channel SNR may become as low as $4 \mathrm{~dB}$ due to deep fades of the channel. On the other hand, we can see that for the STB code $\mathbf{G}_{2}$ using two receivers, the variation in the instantaneous channel SNR becomes significantly less severe.

By employing multiple transmit antennas as shown in Fig. 16, we have reduced the effect of the channels' deep fades significantly. Hence, the advantages of adaptive modulation over fixed-mode modems gradually erode. Therefore, once the fading depth has been sufficiently attenuated, there is no need for employing further fading countermeasures such as adaptive modulation. The lower complexity fixed-mode modems are perfectly adequate in these scenarios. As we increase the number of receivers, i.e., the diversity order, we observe that the variation 

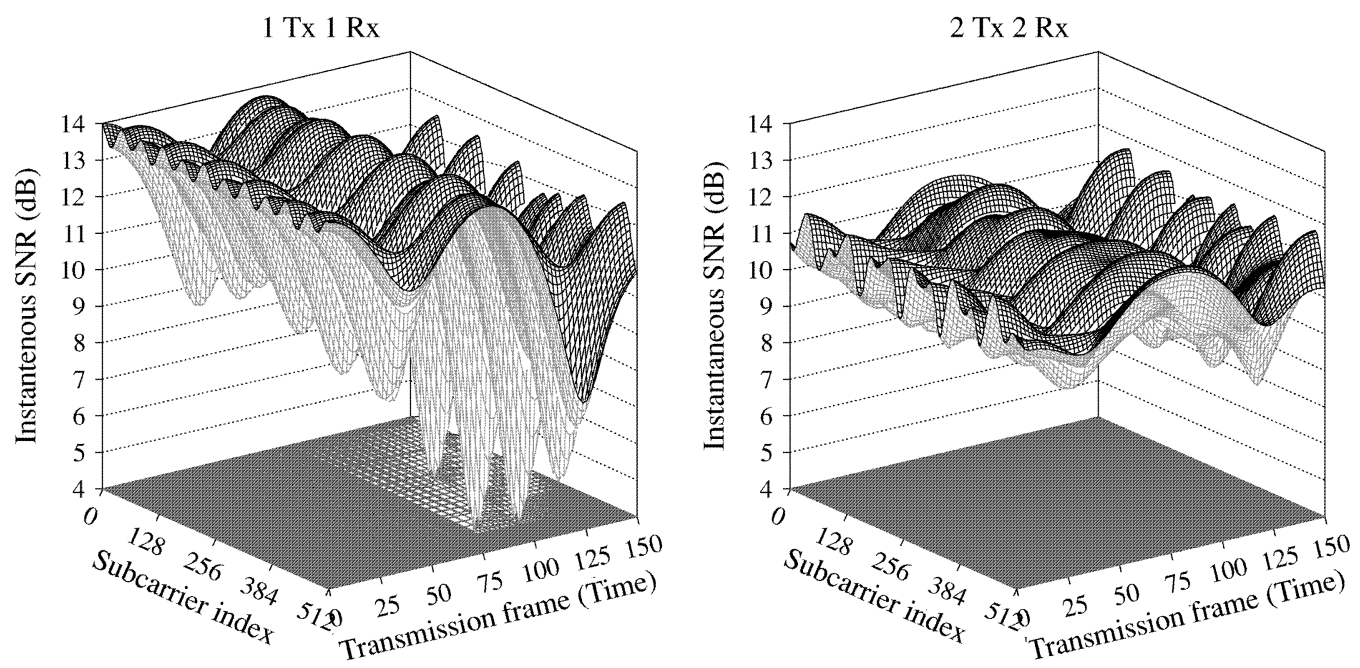

Fig. 16. Instantaneous channel SNR of 512-subcarrier OFDM symbols for single-transmitter single-receiver and for the space-time block code $\mathbf{G}_{2}$ using two receivers over the shortened WATM channel shown in Fig. 14. The average channel SNR is $10 \mathrm{~dB}$.

of the channel magnitude and phase becomes lower. Effectively, by employing higher order diversity, the fading channels have been converted to near-constant magnitude AWGN-like channels, as evidenced by the STB code $\mathbf{G}_{2}$ using two receivers. In conclusion, since adaptive modulation only offers advantages over fading channels, we argue that using adaptive modulation might become unnecessary as the diversity order is increased.

To elaborate further, we surmise from Fig. 16 that fixed modulation schemes might become more attractive when the diversity order increases, which is achieved in this case by employing more receivers. This is because for a certain average channel SNR, the probability of a particular modulation mode increases. In other words, the fading channel has become an AWGN-like channel, as the diversity order is increased. In Fig. 17, we show our throughput performance comparison between AOFDM and fixed modulation-based OFDM in conjunction with the STB code $\mathbf{G}_{2}$ employing 1) one receiver and 2) two receivers over the shortened WATM channel. The throughput of fixed OFDM was $1,2,4$, and 6 BPS, and the corresponding $E_{b} / N_{0}$ values were extracted from the associated BER versus $E_{b} / N_{0}$ curves of the individual fixed-mode OFDM schemes. It can be seen from Fig. 17(a) that the throughput performance of the adaptive and fixed OFDM schemes is similar for a $10^{-2}$ target BER system. However, for a $10^{-4}$ target BER system, there is an improvement of 5-10 dB in $E_{b} / N_{0}$ terms at various throughputs for the adaptive OFDM scheme over the fixed OFDM scheme. At high average channel SNRs, the throughput performance of both schemes converged, since 64QAM became the dominant modulation mode for AOFDM.

On the other hand, if the number of receivers is increased to two, we can see in Fig. 17(b) that the throughput performance of both adaptive and fixed OFDM is similar for both the $10^{-4}$ and $10^{-2}$ target BER systems. We would expect similar trends as the number of receivers is increased, since the fading channels become AWGN-like channels. From Fig. 17, we conclude that AOFDM is only beneficial for the space-time block code $\mathbf{G}_{2}$ using one receiver in the context of the $10^{-2}$ target BER system.
3) Turbo and Space-Time Coded Adaptive OFDM: In the previous section, we have discussed the performance of spacetime coded adaptive OFDM. Here, we extend our study by concatenating turbo coding with the space-time coded AOFDM scheme in order to improve both the BER and BPS performance of the system. As previously seem, the turbo convolutional code $\mathrm{TC}(2,1,3)$ having a constraint length of three and octal generator polynomial of $(7,5)$ was employed. Since the system was designed for high-integrity, low-BER data transmission, it was delay nonsensitive. Hence, a random turbo interleaver size of approximately 10000 bits was employed. The random separation channel interleaver [26] was utilized in order to disperse the bursty channel errors. The Log-MAP [28] decoding algorithm was employed, using eight iterations.

We proposed two TC coded schemes for the space-time coded AOFDM system. The first scheme is a fixed half-rate turbo and space-time coded adaptive OFDM system. It achieves a high BER performance but at the cost of a maximum throughput limited to 3 BPS due to half-rate channel coding. The second one is a variable-rate turbo and space-time coded adaptive OFDM system. This scheme sacrifices the BER performance in exchange for an increased system throughput. Different puncturing patterns are employed for the various code rates $R$. The puncturing patterns were optimized experimentally by simulations. The design procedure for punctured turbo codes was proposed by Acikel et al. [52] in the context of BPSK and QPSK. We emphasize again that the optimum AOFDM mode switching thresholds were obtained for transmission over the shortened WATM channel of Fig. 14 following the AOFDM design procedures of [16] and [17], which are shown in Table VII. As noted previously, more recent research revealed that the optimum switching thresholds are slightly SNRdependent [17], [37], and hence, the thresholds employed here were somewhat suboptimum. Future research is expected to contrive real-time switching threshold control algorithms which do not necessitate the offline optimization of the switching thresholds. 


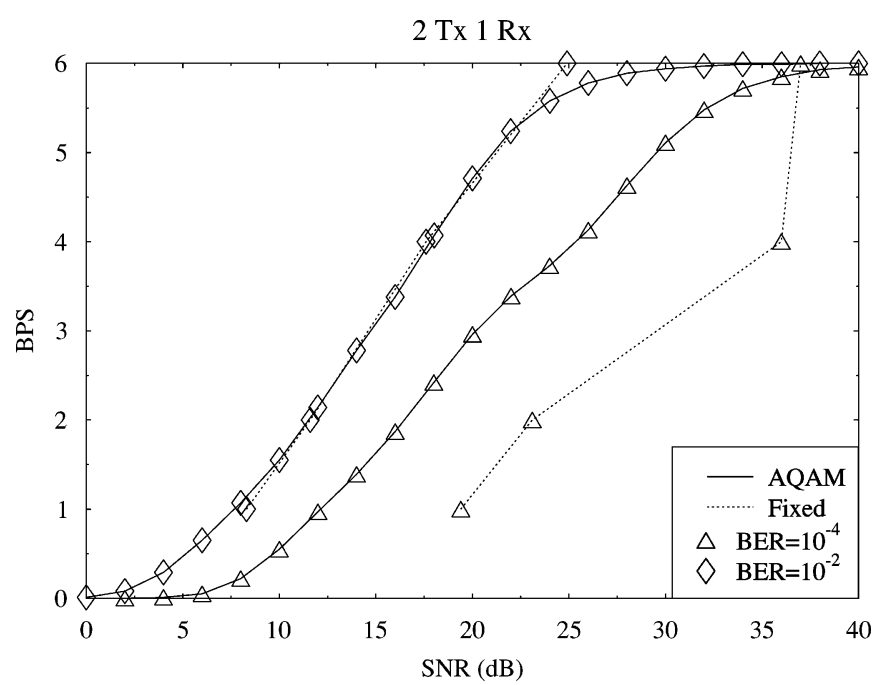

(a)

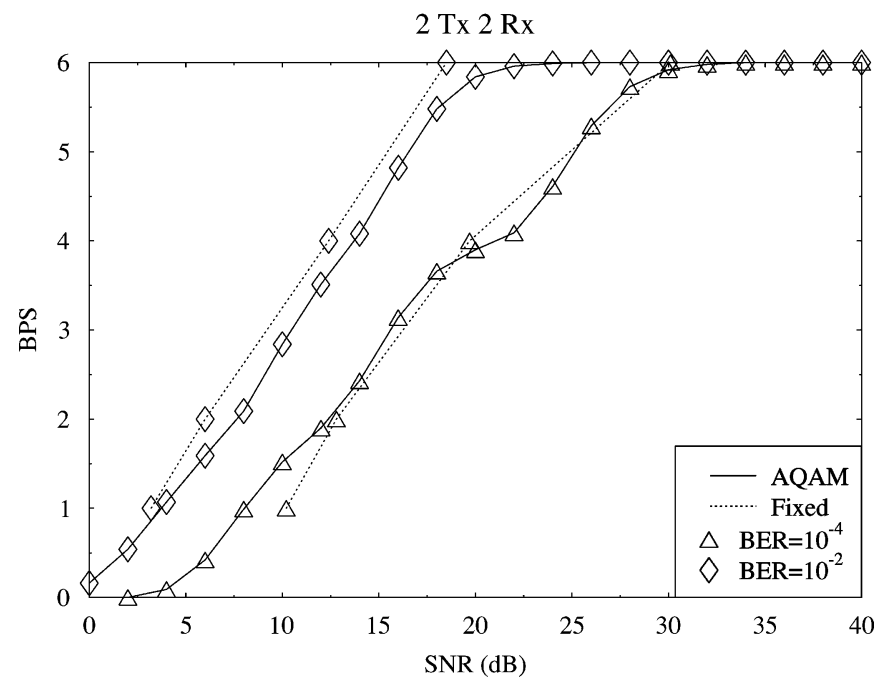

(b)

Fig. 17. BPS throughput performance comparison between adaptive OFDM and fixed modulation-based OFDM using the STB code $\mathbf{G}_{2}$ employing (a) one receiver and (b) two receivers over the shortened WATM channel shown in Fig. 14.

In Fig. 18, we show the BER and BPS performance of the 16-subband AOFDM employing the STB code $\mathbf{G}_{2}$ concatenated with both half-rate and variable-rate $\mathrm{TC}(2,1,3)$ coding at a target BER of $10^{-4}$ over the shortened WATM channel of Fig. 14. We can see in the figure that by concatenating fixed half-rate turbo coding with the space-time coded adaptive OFDM scheme, the BER performance of the system improves tremendously, indicated by a steep dip of the associated BER curve marked by the solid line and diamonds. There is an improvement in the BPS performance as well, exhibiting an $E_{b} / N_{0}$ gain of approximately 5 and $10 \mathrm{~dB}$ at an effective throughput of 1 BPS, compared to space-time coded AOFDM and conventional AOFDM, respectively. However, again, the maximum throughput of the system is limited to $3 \mathrm{BPS}$, since half-rate channel coding was employed. In Fig. 18, we can see that at an $E_{b} / N_{0}$ value of about $30 \mathrm{~dB}$, the maximum
TABLE VII

Coding RATES AND Switching LeVEls (IN DeCiBels) For TC $(2,1,3)$ AND SPACE-Time CODED AdAPTIVE OFDM OVER THE SHORTENED WATM CHANNEL OF FIG. 14 FOR A TARGET BER OF $10^{-4}$

\begin{tabular}{|c|c|c|c|c|c|}
\hline & NoTx & BPSK & QPSK & 16QAM & 64QAM \\
\hline \multicolumn{7}{|c|}{ Half-rate TC $(2,1,3)$} \\
\hline Rate & - & 0.50 & 0.50 & 0.50 & 0.50 \\
\hline Thresholds (dB) & $-\infty$ & -4.0 & -1.3 & 5.4 & 9.8 \\
\hline \multicolumn{7}{|c|}{ Variable-rate TC $(2,1,3)$} \\
\hline Rate & - & 0.50 & 0.67 & 0.75 & 0.90 \\
\hline Thresholds (dB) & $-\infty$ & -4.0 & 2.0 & 9.70 & 21.50 \\
\hline
\end{tabular}

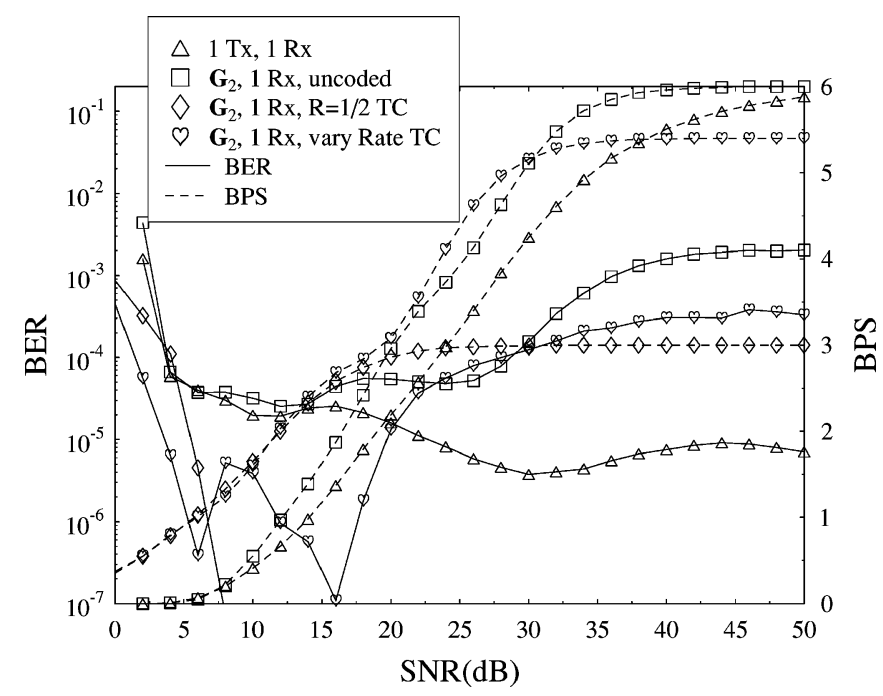

Fig. 18. BER and BPS performance of 16-subband AOFDM employing the STB code $\mathbf{G}_{2}$ concatenated with both half-rate and variable-rate TC $(2,1,3)$ at a target BER of $10^{-4}$ over the shortened WATM channel shown in Fig. 14. The switching thresholds and coding rates are shown in Table VI.

throughput of the turbo coded and space-time adaptive OFDM system is increased from 3.0 to 5.4 BPS by employing the variable-rate $\mathrm{TC}(2,1,3)$ code. Furthermore, the BPS performance of the variable-rate turbo coded scheme is similar to that of the half-rate turbo coded scheme at an average channel SNR below $15 \mathrm{~dB}$. The BER curve marked by the solid line and clubs drops as the average channel SNR is increased from 0 to $15 \mathrm{~dB}$. Due to the increased probability of the 64QAM transmission mode, the variable-rate turbo coded scheme was overloaded by the plethora of channel errors introduced by the 64QAM mode. Therefore, we can see in Fig. 18 that the BER increases and stabilizes at $10^{-4}$. Again, the interference of the signals $x_{1}$ and $x_{2}$ in the context of the STB code $\mathbf{G}_{2}$ prohibits further improvements in the BER performance as the average channel SNR is increased. However, employing the variable-rate turbo codec has lowered the BER floor, as demonstrated by the curve marked by the solid line and squares.

\section{CONCLUSION}

Space-time trellis codes [5]-[11] and space-time block codes [1]-[3], [5] constitute state-of-the-art transmission schemes based on multiple transmitters and receivers. The branch metrics 
of each trellis transition were derived in order to facilitate their maximum likelihood (ML) decoding.

In Section II, we proposed to employ an OFDM modem for mitigating the effects of dispersive multipath channels due to its simplicity compared to other approaches [14], [15]. Turbo codes and RS codes were invoked in Section II-A for concatenation with the STB code $\mathbf{G}_{2}$ and the various STT codes, respectively. The estimated complexity of the various STT codes was derived in Section II-C.

We presented our simulation results for the proposed schemes in Section III. The first scheme studied was the TC $(2,1,3)$ coded STB code $\mathbf{G}_{2}$, whereas the second one was based on the family of STT codes. It was found that the FER and BER performance of the TC $(2,1,3)$ coded STB $\mathbf{G}_{2}$ was better than that of the investigated STT codes at a throughput of 2 and 3 BPS over the channel exhibiting two equal power rays separated by a delay spread of $5 \mu$ s and having a maximum Doppler frequency of 200 Hz. Our comparison between the two schemes was performed by also considering the estimated complexity of both schemes. It was found that the concatenated $\mathbf{G}_{2} / \mathrm{TC}(2,1,3)$ scheme still outperformed the STT codes using no channel coding, even though both schemes exhibited a similar complexity.

Although the corresponding results are not shown here due to space limitations, it was found that the maximum Doppler frequency had no significant impact on the performance of the STB and STT-coded OFDM schemes when mapping the two transmission instants of the STB-coded scheme onto two adjacent OFDM subcarriers. In contrast, in Section III-B, we investigated the effect of the delay spread on the system. Initially, the delay-spread dependent SIR of the STB code $\mathbf{G}_{2}$ was quantified. It was found that the performance of the concatenated TC $(2,1,3)-\mathbf{G}_{2}$ scheme degrades as the delay spread increases due to the decrease in the associated SIR. However, varying the delay spread had no significant effect on the STT codes. We proposed in Section III-C an alternative mapping of the two transmission instants of the STB code $\mathbf{G}_{2}$ to the same subcarrier of two consecutive OFDM symbols: a solution which was applicable to a delay nonsensitive system. By employing this approach, the performance of the concatenated scheme was no longer limited by the delay spread but by the maximum Doppler frequency. We concluded that a certain minimum SIR has to be maintained for attaining the best possible performance of the concatenated scheme.

The shortened WATM channel was introduced in Section IVC1. In this section, STT codes were concatenated with RS codes in order to improve the performance of the system. Once again, both channel-coded STB and STT codes were compared at a throughput of 1 and 2 BPS. It was also found that the TC $(2,1,3)$ coded STB code $\mathbf{G}_{2}$ outperforms the RS-coded STT codes.

Space-time block-coded AOFDM was studied in Section IV. It was shown in Section IV-C2 that only the STB code $\mathbf{G}_{2}$, using one AOFDM receiver, was capable of outperforming the conventional single-transmitter, single-receiver AOFDM system designed for a data transmission target BER of $10^{-4}$ over the shortened WATM channel. We also confirmed that upon increasing the diversity order, the fading channels become AWGN-like channels. This explains why the benefits of AOFDM erode when the diversity order is increased. In Section IV-C3, we continued our investigations into AOFDM by concatenating turbo coding with the system. Two schemes were proposed: half-rate turbo and space-time-coded AOFDM, as well as variable-rate turbo and space-time-coded AOFDM. Despite the impressive BER performance of the halfrate turbo and space-time-coded scheme, the maximum throughput of the system was limited to 3 BPS. However, by employing the variable-rate turbo and space-time coded scheme, the BPS performance improved, achieving a maximum throughput of 5.4 BPS. This BPS performance improvement was achieved at the cost of a poorer BER performance. In conclusion, this is a burgeoning research area, which is expected to achieve further advances in the field of turbo space-time coding [5].

\section{REFERENCES}

[1] S. Alamouti, "A simple transmit diversity technique for wireless communications,” IEEE J. Sel. Areas Commun., vol. 16, no. 8, pp. 1451-1458, Oct. 1998.

[2] V. Tarokh, H. Jafarkhani, and A. Calderbank, "Space-time block codes from orthogonal designs," IEEE Trans. Inf. Theory, vol. 45, no. 5, pp. 1456-1467, Jul. 1999.

[3] V. Tarokh, H. Jafarkhani, and A. Calderbank, "Space-time block coding for wireless communications: performance results," IEEE J. Sel. Areas Commun., vol. 17, no. 3, pp. 451-460, Mar. 1999.

[4] T. Liew and L. Hanzo, "Space-time block codes and concatenated channel codes for wireless communications," Proc. IEEE, vol. 90, no. 2, pp. 183219, Feb. 2002.

[5] L. Hanzo, T. Liew, and B. Yeap, Turbo Coding, Turbo Equalisation, and Space-Time Coding. Piscataway, NJ: Wiley, IEEE Press, 2002.

[6] V. Tarokh, N. Seshadri, and A. Calderbank, "Space-time codes for high data rate wireless communication: performance criterion and code construction," IEEE Trans. Inf. Theory, vol. 44, no. 2, pp. 744-765, Mar. 1998.

[7] N. Seshadri, V. Tarokh, and A. Calderbank, "Space-time codes for high data rate wireless communications: code construction," in Proc. IEEE Vehicular Technology Conf:'97, Phoenix, AZ, 1997, pp. 637-641.

[8] V. Tarokh, N. Seshadri, and A. Calderbank, "Space-time codes for high data rate wireless communications: performance criterion and code construction," in Proc. IEEE Int. Conf. Communications'97, Montreal, QC, Canada, 1997, pp. 299-303.

[9] V. Tarokh, A. Naguib, N. Seshadri, and A. Calderbank, "Space-time codes for high data rate wireless communications: mismatch analysis," in Proc. IEEE Inter. Conf. Communications' 97, Montreal, QC, Canada, 1997, pp. 309-313.

[10] A. Naguib, V. Tarokh, N. Seshadri, and A. Calderbank, "A space-time coding modem for high-data-rate wireless communications," IEEE J. Sel. Areas Commun., vol. 16, no. 8, pp. 1459-1478, Oct. 1998.

[11] V. Tarokh, A. Naguib, N. Seshadri, and A. Calderbank, "Space-time codes for high data rate wireless communication: performance criteria in the presence of channel estimation errors, mobility, and multiple paths," IEEE Trans. Commun., vol. 47, no. 2, pp. 199-207, Feb. 1999.

[12] R. Horn and C. Johnson, Matrix Analysis. Cambridge, U.K.: Cambridge Univ. Press, 1988.

[13] A. Naguib, N. Seshadri, and A. Calderbank, "Increasing data rate over wireless channels," IEEE Signal Process. Mag., vol. 17, no. 5, pp. 76-92, May 2000.

[14] G. Bauch, A. Naguib, and N. Seshadri, "MAP equalization of spacetime coded signals over frequency selective channels," in Proc. Wireless Communications and Networking Conf., New Orleans, LA, Sep. 1999.

[15] G. Bauch and N. Al-Dhahir, "Reduced-complexity turbo equalization with multiple transmit and receive antennas over multipath fading channels," in Proc. Information Sciences and Systems, Princeton, NJ, Mar. 2000, pp. WP3 13-18.

[16] L. Hanzo, W. Webb, and T. Keller, Eds., Single- and Multi-Carrier Quadrature Amplitude Modulation, 3rd ed.. New York: Wiley, 2000.

[17] L. Hanzo, M. Münster, B.-J. Choi, and T. Keller, OFDM and MC-CDMA for Broadband Multi-User Communications, WLANs and Broadcasting. Piscataway, NJ: IEEE Press, Wiley, 2003. 
[18] D. Agrawal, V. Tarokh, A. Naguib, and N. Seshadri, "Space-time coded OFDM for high data-rate wireless communication over wideband channels," in Proc. IEEE Vehicular Technology Conf., Ottawa, ON, Canada, May 1998, pp. 2232-2236.

[19] Y. Li, N. Seshadri, and S. Ariyavisitakul, "Channel estimation for OFDM systems with transmitter diversity in mobile wireless channels," IEEE $J$. Sel. Areas Commun., vol. 17, no. 3, pp. 461-471, Mar. 1999.

[20] Y. Li, J. Chuang, and N. Sollenberger, "Transmitter diversity for OFDM systems and its impact on high-rate data wireless networks," IEEE J. Sel. Areas Commun., vol. 17, no. 7, pp. 1233-1243, Jul. 1999.

[21] W.-J. Choi and J. Cioffi, "Space-time block codes over frequency selective fading channels," in Proc. Vehicular Technology Conf., Amsterdam, The Netherlands, Fall 1999, pp. 2541-2545.

[22] Z. Liu, G. Giannakis, A. Scaglione, and S. Barbarossa, "Block precoding and transmit-antenna diversity for decoding and equalization of unknown multipath channels," in Proc. 33rd Asilomar Conf. Signals, Systems and Computers, Pacific Grove, CA, Nov. 1999, pp. 1557-1561.

[23] Z. Liu and G. Giannakis, "Space-time coding with transmit antennas for multiple access regardless of frequency-selective multipath," in Proc 1st Sensor Array and Multichannel SP Workshop, Boston, MA, Mar. 2000.

[24] T. Liew, J. Pliquett, B. Yeap, L.-L. Yang, and L. Hanzo, "Concatenated space time block codes and TCM, turbo TCM, convolutional as well as turbo codes," in Proc. GLOBECOM 2000, San Francisco, CA.

[25] R. Steele and L. Hanzo, Mobile Radio Communications. New York: Wiley, 1999.

[26] T. Liew, J. Pliquett, B. Yeap, L.-L. Yang, and L. Hanzo, "Comparative study of space time block codes and various concatenated turbo coding schemes," in Proc. PIMRC 2000, London, U.K., Sep. 2000, pp. 741-745.

[27] L. Bahl, J. Cocke, F. Jelinek, and J. Raviv, "Optimal decoding of linear codes for minimizing symbol error rate," IEEE Trans. Inf. Theory, vol. IT20, no. 2, pp. 284-287, Mar. 1974.

[28] P. Robertson, E. Villebrun, and P. Hoeher, "A comparison of optimal and sub-optimal MAP decoding algorithms operating in the log domain," in Proc. Int. Conf. Communications, Jun. 1995, pp. 1009-1013.

[29] G. Bauch, "Concatenation of space-time block codes and turbo-TCM," in Proc. IEEE Int. Conf. Communications, Vancouver, BC, Canada, Jun. 1999, pp. 1202-1206

[30] G. Forney, "The Viterbi algorithm," Proc. IEEE, vol. 61, pp. 268-277, Mar. 1973.

[31] L.-L. Yang and L. Hanzo, "Performance analysis of $m$-ary orthogonal signaling using errors-and-erasures decoding over frequency-selective Rayleigh fading channels," IEEE J. Sel. Areas Commun., vol. 19, no. 2, pp. 211-221, Feb. 2001.

[32] W. Jakes, Microwave Mobile Communications. Piscataway, NJ: IEEE Press, 1993

[33] L. Hanzo, F. Somerville, and J. Woodard, Voice Compression and Communications: Principles and Applications for Fixed and Wireless Channels. Piscataway, NJ: IEEE Press, Wiley, 2001.

[34] L. Hanzo, P. Cherriman, and J. Streit, Wireless Video Communications: From Second to Third Generation Systems, WLANs and Beyond. Piscataway, NJ: IEEE Press, Wiley, 2001.

[35] R. Steele and W. Webb, "Variable rate QAM for data transmission over Rayleigh fading channels," in Proc. Wireless'91, Calgary, AB, Canada, 1991, pp. 1-14.

[36] W. Webb and R. Steele, "Variable rate QAM for mobile radio," IEEE Trans. Commun., vol. 43, no. 7, pp. 2223-2230, Jul. 1995.

[37] L. Hanzo, C. Wong, and M. Yee, Adaptive Wireless Transceivers. Piscataway, NJ: IEEE Press, Wiley, 2002.

[38] J. Torrance and L. Hanzo, "Latency and networking aspects of adaptive modems over slow indoors Rayleigh fading channels," IEEE Trans. Veh. Technol., vol. 48, no. 4, pp. 1237-1251, Jul. 1999.

[39] J. Torrance and L. Hanzo, "Performance upper bound of adaptive QAM in slow Rayleigh fading environments," in Proc. ISPACS' 96, Singapore, 1996, pp. 1653-1657.

[40] J. Torrance and L. Hanzo, "Interference aspects of adaptive modems over slow Rayleigh fading channels," IEEE Trans. Veh. Technol., vol. 48, no. 5, pp. 1527-1545, Sep. 1999.

[41] T. Keller and L. Hanzo, "Adaptive orthogonal frequency division multiplexing schemes," in Proc. ACTS Mobile Communications Summit'98, Rhodos, Greece, Jun. 1998, pp. 794-799.
[42] T. Keller and L. Hanzo, "Blind-detection assisted sub-band adaptive turbocoded OFDM schemes," in Proc. Vehicular Technology Conf. VTC'99, Houston, TX, 1999, pp. 127-130.

[43] H. Matsuako, S. Sampei, N. Morinaga, and Y. Kamio, "Adaptive modulation systems with variable coding rate concatenated code for high quality multi-media communication systems," in Proc. IEEE Vehicular Technology Conf., Atlanta, GA, 1996, pp. 487-491.

[44] S.-G. Chua and A. Goldsmith, "Variable-rate variable-power mQAM for fading channels," in Proc. IEEE Vehicular Technology Conf.'96, Atlanta, MA, 1996, pp. 815-819.

[45] V. Lau and M. Macleod, "Variable rate trellis coded QAM for high bandwidth efficiency applications in Rayleigh fading channels," in Proc. IEEE Vehicular Technology Conf.' 98, Ottawa, ON, Canada, 1998, pp. 348-352.

[46] J. Blogh and L. Hanzo, $3 G$ Systems and Intelligent Networking. Piscataway, NJ: IEEE Press, Wiley, 2002.

[47] L. Hanzo, L. L. Yang, E. L. Kuan, and K. Yen, Single- and Multi-Carrier CDMA. Piscataway, NJ: IEEE Press, Wiley, 2003.

[48] I. Kalet, "The multitone channel," IEEE Trans. Commun., vol. 37, no. 2, pp. 119-124, Feb. 1989.

[49] T. Keller and L. Hanzo, "Adaptive multicarrier modulation: A convenient framework for time-frequency processing in wireless communications," Proc. IEEE, vol. 88, no. 5, pp. 611-642, May 2000.

[50] J. Torrance and L. Hanzo, "Optimization of switching levels for adaptive modulation in a slow Rayleigh fading channel," Electron. Lett., vol. 32, no. 13, pp. 1167-1169, Jun. 1996.

[51] J. Torrance and L. Hanzo, "On the upper bound performance of adaptive QAM in a slow Rayleigh fading," Electron. Lett.,pp. 169-171, Apr. 1996.

[52] O. Acikel and W. Ryan, "Punctured turbo-codes for BPSK/QPSK channels," IEEE Trans. Commun., vol. 47, no. 9, pp. 1315-1323, Sep. 1999.

Tong-Hooi Liew received the B.Eng. degree in electronics engineering and the Ph.D. degree from the University of Southampton, Southampton, U.K., in 2001. $\mathrm{He}$ is currently continuing his research as a Postdoctoral Research Fellow at the University of Southampton.

His current research interests are associated with coding and modulation for wireless channels, space-time coding, and adaptive transceivers. He has published his research results widely, including coauthoring a Wiley-IEEE Press monograph.

Lajos Hanzo (F'02) received the M.S. degree in electronics in 1976, the Ph.D. degree in 1983, and the D.Sc. degree from the University of Southampton, Southampton, U.K., in 2004.

During his 28-year career in telecommunications, he has held various research and academic posts in Hungary, Germany, and the U.K. Since 1986, he has been with the Department of Electronics and Computer Science, University of Southampton, U.K., where he holds the Chair in telecommunications. He has co-authored 11 Wiley/IEEE Press books totaling about 9000 pages on mobile radio communications, published in excess of 500 research papers, organized and chaired conference sessions, presented overview lectures, and has been awarded numerous distinctions. Currently, he is managing an academic research team and working on a range of research projects in the field of wireless multimedia communications sponsored by industry, the Engineering and Physical Sciences Research Council (EPSRC) U.K., and the European IST Programme, and the Mobile Virtual Centre of Excellence (VCE), U.K. He is an enthusiastic supporter of industrial and academic liaison, and he offers a range of industrial courses.

Dr. Hanzo is an IEEE Distinguished Lecturer of both the IEEE Communications and the Vehicular Technology Societies and a Fellow of the IEE and the RoyalAcademy of Engineering (FREng). 